\title{
ISOLATION OF FREE-LIVING NITROGEN-FIXING BACTERIA FROM THE RHIZOSPHERE OF RICE
}

\author{
YAEKO OYAIZU-MASUCHI AND KAZUO KOMAGATA \\ Institute of Applied Microbiology, The University of Tokyo, \\ Bunkyo-ku, Tokyo 113, Japan
}

(Received January 25, 1988)

\begin{abstract}
Bacterial flora of rhizosphere of the rice plant was examined to determine the relationship of nitrogen-fixing bacteria to rice plants. Of 7,344 bacterial strains from the rhizosphere, 1,005 strains $(13.7 \%)$ reduced acetylene. Representative 74 strains with acetylene reduction activity were selected for identification. All of these strains were gram negative, aerobic or facultatively anaerobic, and most had ubiquinone (Q-10) and cellular fatty acid composition, mainly with straight-chain fatty acids. Twenty-two strains were assigned to the following known species: Azospirillum brasilense Tarrand, Krieg and Döbereiner (1 strain), Azospirillum lipoferum Tarrand, Krieg and Döbereiner (1 strain), Enterobacter cloacae (Jordon) Hormaeche and Edwards (4 strains), Erwinia herbicola (Löhnis) Dye (2 strains), Klebsiella oxytoca (Flugge) Lautrop (6 strains), and Xanthobacter autotrophicus (Baumgarten, Reh and Schlegel) Wiegel, Wilke, Baumgarten, Opitz and Schlegel ( 8 strains). Fifty-two strains could not be assigned to any known species.
\end{abstract}

Since Yoshida and AnCAJAs ( 1 ) reported the association of free-living nitrogen-fixing bacteria with rice plants, several workers have isolated such bacteria and investigated the interrelation between bacteria and rice (2-17). Until recently, Azospirillum lipoferum(11-14), Azospirillium brasilense (12-14), Enterobacter cloacae $(12,13,15)$, Klebsiella oxytoca $(12,13)$, Pseudomonas paucimobilis $(12,13)$, Pseudomonas sp. (16,17), and Clostridium sp. (5) have been isolated. SANo et al.(18) reported a seasonal variation of nitrogen-fixation in the rice plant and a possible symbiotic relation between bacteria and the plants. We have collaborated with them to investigate the relation between the nitrogen-fixing bacteria and the rice plant.

This paper deals with the characterization and identification of the free-living nitrogen-fixing bacteria isolated from rice roots and paddy soil.

Address reprint requests to: Dr. K. Komagata, Institute of Applied Microbiology, The University of Tokyo, Yayoi 1-1-1, Bunkyo-ku, Tokyo 113, Japan. 


\section{MATERIALS AND METHODS}

Materials. Plant materials of Oryza sativa strains C5444 and T65 and paddy soils were surveyed as isolation sources of free-living nitrogen-fixing bacteria. These materials were obtained from the National Institute of Genetics, Mishima, Japan during 3 years (1980-1982) by the courtesy of Dr. Fujii. Rice plants were grown in a greenhouse in plastic pots $(7.2 \mathrm{~cm}$ diam.). Each pot contained $180 \mathrm{~g}$ of soil $(\mathrm{pH}$ 6.5 ) and fertilizers (urea $0.043 \mathrm{~g}$, superphosphate of lime $0.267 \mathrm{~g}, \mathrm{KCl} 0.04 \mathrm{~g}$, rotted soil $2 \mathrm{~g}$ ).

Isolation medium. Three kinds of media were used for isolation: 1) The malate medium reported by VON BULOW and DöBEREINER (19) was composed of $0.4 \mathrm{~g}$ of $\mathrm{KH}_{2} \mathrm{PO}_{4}, 0.2 \mathrm{~g}$ of $\mathrm{MgSO}_{4} \cdot 7 \mathrm{H}_{2} \mathrm{O}, 0.1 \mathrm{~g}$ of $\mathrm{NaCl}, 10 \mathrm{mg}$ of $\mathrm{FeCl}_{3}, 2 \mathrm{mg}$ of $\mathrm{Na}_{2} \mathrm{MoO}_{4} \cdot 2 \mathrm{H}_{2} \mathrm{O}, 0.1 \mathrm{~g}$ yeast extract, $2 \mathrm{ng}$ of biotin, and $1,000 \mathrm{ml}$ of distilled water. The $\mathrm{pH}$ of the medium was adjusted with $\mathrm{KOH}$ to 6.8 , and Bromothymol Blue (BTB) was used as an indicator. This medium was designated as M medium. 2) The nitrogen-free glucose medium of STANIER et al. (20) was composed of $10 \mathrm{~g}$ of glucose, $0.2 \mathrm{~g}$ of $\mathrm{MgSO}_{4} \cdot 7 \mathrm{H}_{2} \mathrm{O}, 1.0 \mathrm{~g}$ of $\mathrm{K}_{2} \mathrm{HPO}_{4}, 50 \mathrm{mg}$ of $\mathrm{FeSO}_{4} \cdot 7 \mathrm{H}_{2} \mathrm{O}, 20 \mathrm{mg}$ of $\mathrm{CaCl}_{2}, 2 \mathrm{mg}$ of $\mathrm{MnSO}_{4} \cdot 4-6 \mathrm{H}_{2} \mathrm{O}, 1 \mathrm{mg}$ of $\mathrm{NaMoO}_{4} \cdot 2 \mathrm{H}_{2} \mathrm{O}, 5 \mathrm{~g}$ of $\mathrm{CaCO}_{3}, 0.1 \mathrm{~g}$ of yeast extract, and $2 \mathrm{ng}$ of biotin, and $1,000 \mathrm{ml}$ of distilled water. The $\mathrm{pH}$ of the medium was adjusted with $\mathrm{NaOH}$ to 7.0. The $\mathrm{CaCO}_{3}$ was heat-sterilized separately. This medium was designated as NFG medium. 3) The nutrient medium was composed of $10 \mathrm{~g}$ of beef extract (Difco Laboratories, Michigan, U.S.A.), $10 \mathrm{~g}$ of Polypepton (Daigo, Osaka, Japan), $5 \mathrm{~g}$ of $\mathrm{NaCl}$, and 1,000 $\mathrm{ml}$ of distilled water. The $\mathrm{pH}$ of the medium was adjusted with $\mathrm{NaOH}$ to 7.0. Two grams of agar were added to $1,000 \mathrm{ml}$ of medium to prepare a semisolid medium and $16 \mathrm{~g}$ for a solid medium.

Isolation procedure. (1) Paddy soil: One gram of paddy soil was suspended in $10 \mathrm{ml}$ of sterile water, and $0.1 \mathrm{ml}$ of the suspension was spread on NFG agar plate. The plate was incubated for 7 days at $30^{\circ} \mathrm{C}$ anaerobically using the Gaspack anaerobic system (BBL, Cockeysville, U.S.A.). Colonies from each plate were picked up on agar slant with the same composition and cultivated at $30^{\circ} \mathrm{C}$ aerobically and anaerobically, then acetylene activity (AR activity) was tested. (2) Rice materials: For the isolation from rice plants, rice stubbles were taken out of the pots, and soil adhering to the roots was removed by gently shaking in tap water. Then the roots were washed three times with $200 \mathrm{ml}$ each of sterile water. One gram of the root was ground in $9 \mathrm{ml}$ of sterile water in a mortar with pestle, and a series of 10 -fold dilutions was prepared. From these samples, $0.1 \mathrm{ml}$ was spread on M, NFG, and nutrient agar plates. The plates were incubated aerobically for 5 days at $30^{\circ} \mathrm{C}$ or anaerobically for 7 days using the Gaspack anaerobic system (BBL). Colonies from each plate were picked up on agar slants with the same composition, and the AR activity of isolates was tested. Purified isolates were maintained on nutrient agar slants.

Assay of acetylene reduction. A culture grown on nutrient agar was stabbed into both M and NFG semisolid medium test tubes. After 2 days of incubation at 
$30^{\circ} \mathrm{C}$, a paper stopper was replaced with a tight rubber stopper, then $10 \%$ of the air in the test tube was replaced with acetylene. In the NFG test tube, air was replaced with argon first, and then $10 \%$ of the argon was replaced with acetylene. After $24 \mathrm{~h}$ of incubation at $30^{\circ} \mathrm{C}$, ethylene formed by reduction of acetylene was detected by gas chromatography (Shimadzu GC-mini 2, Shimadzu, Kyoto, Japan) with an $\mathrm{H}_{2}$ flame ionization detector, using 1.8-m column containing Porapak N (80-100 mesh) and $\mathrm{N}_{2}$ gas as the carrier. These gas compositions used to assay acetylene reduction were the best composition for the most effective selection of nitrogen-fixing bacteria.

Identification methods. From the isolates 74 strains with high AR activities were selected for identification. Cell morphology, gram reaction, motility, and flagellar morphology were investigated in cells grown on nutrient agar or $\mathbf{M}$ semisolid agar at 30 or $37^{\circ} \mathrm{C}$ after one day's incubation. Gram staining was carried out by HUCKER and CONN's modification (21). Motility was tested by the hanging drop, and flagellar morphology was determined by the staining method of TODA (22). In addition, preparations negatively stained with a $1 \%$ solution of phosphotungstic acid were examined by electron microscopy. Cell wall and cell shape were also studied by the electron microscopy. The fixative used was a $3 \%$ glutaraldehyde solution in potassium phosphate buffer $(\mathrm{pH} 7.0)$. Fixed cells were washed several times in the buffer and postfixed in $2 \% \mathrm{OsO}_{4}$ for $2 \mathrm{~h}$ at room temperature. After the cells were washed with distilled water, they were stained in $0.5 \%$ aqueous uranyl acetate for $2 \mathrm{~h}$ and embedded in agar blocks. They were dehydrated by passage through a series of increasing concentrations of ethanol and absolute acetone, and embedded in SPURR's resin(23). Sections were cut with a Du Pont diamond knife on a Sorvall MT-2 ultramicrotome (Du Pont Co., Wilmington, Delaware, U.S.A.). They were stained with uranyl acetate and lead citrate(24), then viewed in a JEOL 200CX electron microscope (JEOL, Akishima, Japan) at $100 \mathrm{kV}$. Poly- $\beta$-hydroxybutyrate (PHB) granules were studied by the methods of Burdon (25) and Ostle and Holt (26). Spore formation was investigated by the method described by Cowan (27). Cyst formation was investigated by the method of STEVENSON and Socolofsky (28).

Biochemical characters were investigated basically according to the methods described by CowAN (27) and TARRAND et al. (29). Nutrient agar was used for preculture and as a basal medium. Bacterial strains were cultivated at 30 or $37^{\circ} \mathrm{C}$ without shaking. Production of fluorescent pigments was tested on King A and B media (Nissui Seiyaku, Tokyo, Japan) and mannitol yeast-extract medium(27). Production of pigment from gluconate was investigated on the medium described by KorTH et al. (30). Oxidation and fermentation (OF) basal medium (Difco 0688) was used to determine the production of acid from sugars and sugar alcohols. For spirals, the medium of TARRAND et al.(29) was used. Hydrolysis of esculin was tested as described by COWAN (27) and HYLEMON et al. (31). Hydrolysis of arginine was tested as described by THORNLEY (32). Nitrate reduction was tested in nitrate broth and succinate-nitrate broth (33) after 1, 3, and 7 days' incubation. Nitrate respiration was tested by the method of IIzUKa and Komagata (34). Production of 
hydrogen sulfide was tested on TSI agar (Nissui Seiyaku) for 2 weeks. Tetrazolium chloride reduction was investigated by the method described by SAKAZAKI (35). Christensen medium (Nissui Seiyaku) and Simmons medium (Nissui Seiyaku) were used for the citrate utilization. Malonate medium (Nissui Seiyaku) was used for the malonate utilization and phenylalanine deaminase. Decarboxylase base (Difco 0890) was used to examine lysine and ornithine decarboxylases and arginine dihydrolase. Cytochrome-oxidase reagent-"Nissui" (Nissui Seiyaku) was used to detect oxidase. Extracellular DNase activity was determined on DNase test agar (Difco 0632) plates after 1, 3, and 7 days' incubation. For spirals, urease was investigated by the method of TARRAND et al. (29). Production of 3-ketolactose from lactose was examined as described by Bernaerts and De Ley (36). Assimilation of carbon compounds was tested according to the method described by STANIER et al. (37) and UraKami and Komagata (38) at $30^{\circ} \mathrm{C}$ for 3 weeks with some modification. Carbohydrates, organic acids, and alcohols were used at a concentration of $0.2 \%$ or $1 \%$, but formaldehyde, formamide, and phenol were used at $0.02 \%$ because of their toxicity. Assimilation of polygalacturonate and pectin was tested according to the method of EDwARDS and EwING (39). Utilization of hydrogen was tested by growth under an atmosphere of hydrogen, oxygen, and carbon dioxide $(85: 5: 10)$ in $\mathrm{N}$-free mineral medium (40).

DNA base composition. DNAs were isolated by the method of SAITO and MiUra $(41)$ and the $\mathrm{mol} \%$ guanine + cytosine $(\mathrm{G}+\mathrm{C})$ values were calculated from the thermal denaturation temperature of DNA $\left(T_{\mathrm{m}}\right)$ by the formula of MARMUR and Doty(42). Reference DNA was extracted from Escherichia coli IAM 1268 (=ATCC $11303=$ strain B), and its DNA base composition was presumed to be $50.9 \%$.

$D N A-D N A$ hybridization. Purified DNA was labeled with $\left(1^{\prime}, 2^{\prime}, 5-{ }^{3} \mathrm{H}\right)$ cytidine 5 '-triphosphate in vitro by the nick translation technique described by RIGBY et al. (43), using the ${ }^{3} \mathrm{H}$-Nick Translation kit (Code TRK 625 and Code N5000, Amersham International plc, Buckinghamshire, England). The DNA-DNA hybridization was carried out by the procedure described by KANEKO(44). The radioactivity was determined with a PACKARD TRI-CARB 300 model scintillation spectrometer (Packard Instrument Co., Downers Grove, Illinois, U.S.A.). The relative binding was calculated as follows: Relative binding $(\%)=((\mathrm{cpm}$ of heterologous filters)/(cpm of homologous filters) $) \times 100$.

Quinone system. The quinone system was determined by the method of YAMADA et al. (45).

Cellular fatty acid composition and sphingolipid. The cellular fatty acid composition was determined by the method of Iкемото et al. (46). Cells cultivated in nutrient broth at $30^{\circ} \mathrm{C}$ for $24 \mathrm{~h}$ with shaking were used to analyze cellular fatty acids. Sphingolipid was determined by the method described by Yамамото et al. (47).

Visible absorption spectrum of yellow-pigment. Yellow-pigment was extract from bacterial cells by the method described by YABUUCHI et al. (48). The visible 
absorption spectrum of yellow-pigment in acetone-extract was determined with a Shimadzu Recording Spectrophotometer Model A300 (Shimadzu, Kyoto, Japan).

Reference strains. Reference strains used in this study are listed below. The superscript " $T$ " indicates the type strain of the species. The abbreviations for culture collections were as follows: ATCC, American Type Culture Collection, Rockville, Maryland, U.S.A.; CDC, Centers for Disease Control, Atlanta, Georgia, U.S.A.; DSM, Deutsche Sammlung von Mikroorganismen, Göttingen, West Germany; IAM, Institute of Applied Microbiology, The University of Tokyo, Tokyo, Japan; KM, Kansai Medical School, Osaka, Japan; NCIB, National Collection of Industrial Bacteria, Aberdeen, U. K. Agrobacterium radiobacter IAM $12048^{\mathrm{T}}$, Agrobacterium tumefaciens IAM 1037, Alcaligenes denitrificans IAM 12370, Alcaligenes eutrophus IAM $12368^{\mathrm{T}}$, Alcaligenes faecalis IAM 12369, Alcaligenes latus IAM 12599, Alcaligenes paradoxus DSM 30034, Azomonas agilis DSM 375 Azotobacter chroococcum IAM $12666^{\mathrm{T}}$, Azotobacter vinerandii DSM $87^{\mathrm{T}}$, Azospirillum brasilense IAM $12400^{\mathrm{T}}$, Azospirillum lipoferum IAM $12399^{\mathrm{T}}$, Beijerinkia indica NCIB 8712 ${ }^{\mathrm{T}}$, Derxia gummosa $\mathrm{NCIB} 9064^{\mathrm{T}}$, Enterobacter cloacae IAM 12349, Erwinia herbicola IAM 1562 and IAM 1584, Klebsiella oxytoca RH 1586 (=ATCC 13182), Klebsiella pneumoniae CDC 2551-63 and IAM 12351 ${ }^{\mathrm{T}}$, Protomonas extroquens TK $0001^{\mathrm{T}}$ (= NCIB 9399), Pseudomonas paucimobilis KM $2395^{\mathrm{T}}$ and KM 2397, Pseudomonas sp. strain H3 (17), Xanthobacter autotrophicus DSM 431 and $432^{\mathrm{T}}$, and Xanthobacter flavus NCIB $10071^{\mathrm{T}}$.

\section{RESULTS AND DISCUSSION}

Grouping and identification of isolates

Of the 7,344 isolates from rice materials and paddy soil, 1,005 strains showed AR activity. From these strains 74 strains with high AR activity were selected for identification. The strains studied were divided into 10 groups by morphological, biochemical, and chemotaxonomic characters as shown in Tables 1 and 2 .

Group 1. Azospirilla

Azospirillum brasilense Tarrand, Krieg and Döbereiner 1979

Strain: SL-6

The strain was gram negative and formed spiral or vibrio-like cells. The strain had a polar flagellum and exhibited high spirillum-like motility. Cells were $1.0 \mu \mathrm{m}$ by 1.5 to $5.0 \mu \mathrm{m}$. Old cells became longer than $10 \mu \mathrm{m}$. PHB granules were accumulated in cells. Colonies on nutrient agar plate were white and dull. On semisolid medium cultures they produced whitish rings $1-2 \mathrm{~cm}$ below the surface.

Strain SL-6 did not produce acid from glucose aerobically or anaerobically and did not use glucose as a sole carbon source determined by the method of TARRAND et al. (29). Strain SL-6 did not produce acid from carbon compounds except ribose and fructose as shown in Table 3. Strain SL-6 did not require biotin.

The chemotaxonomic characters of strain SL-6 are shown in Table 2. Strain 


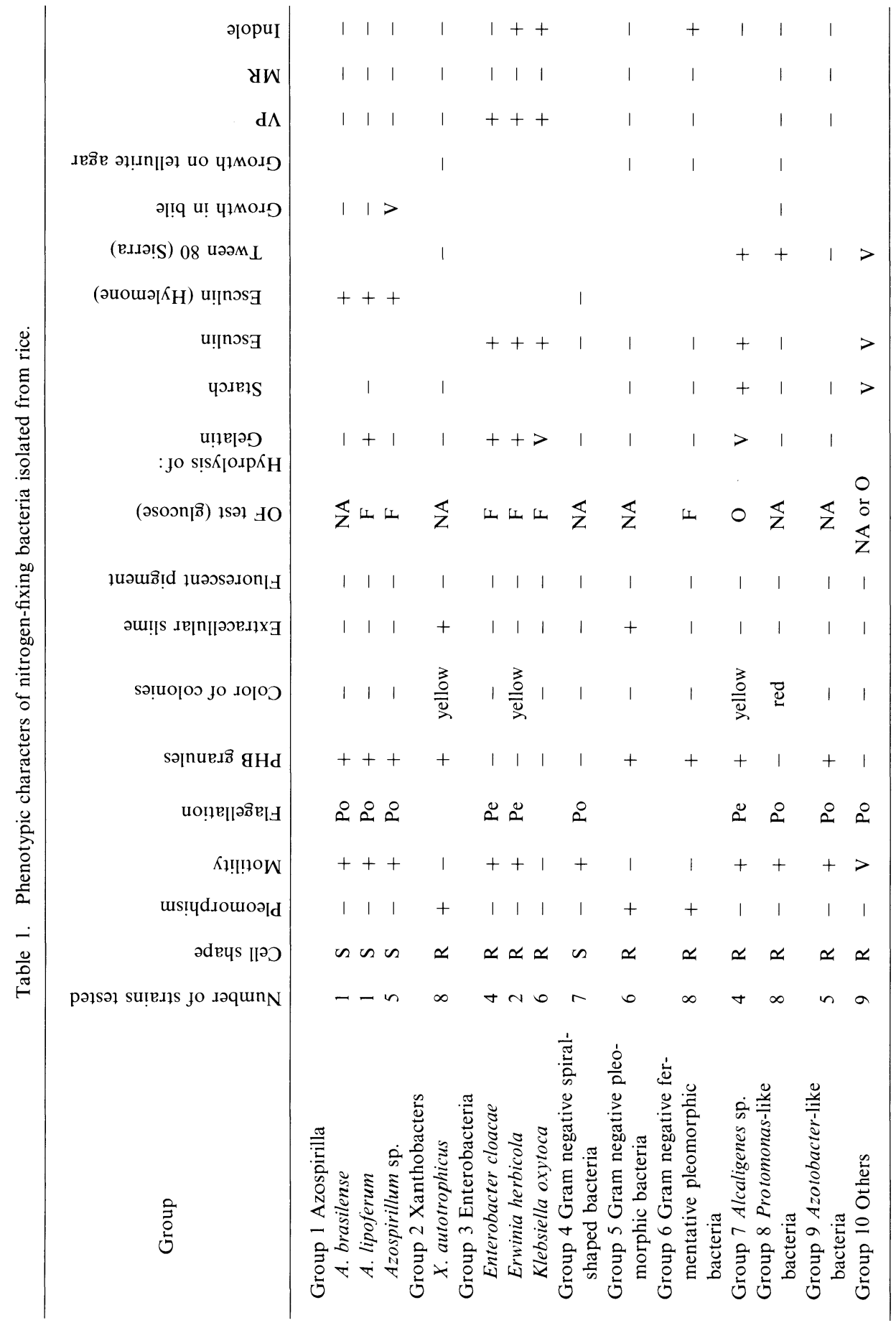




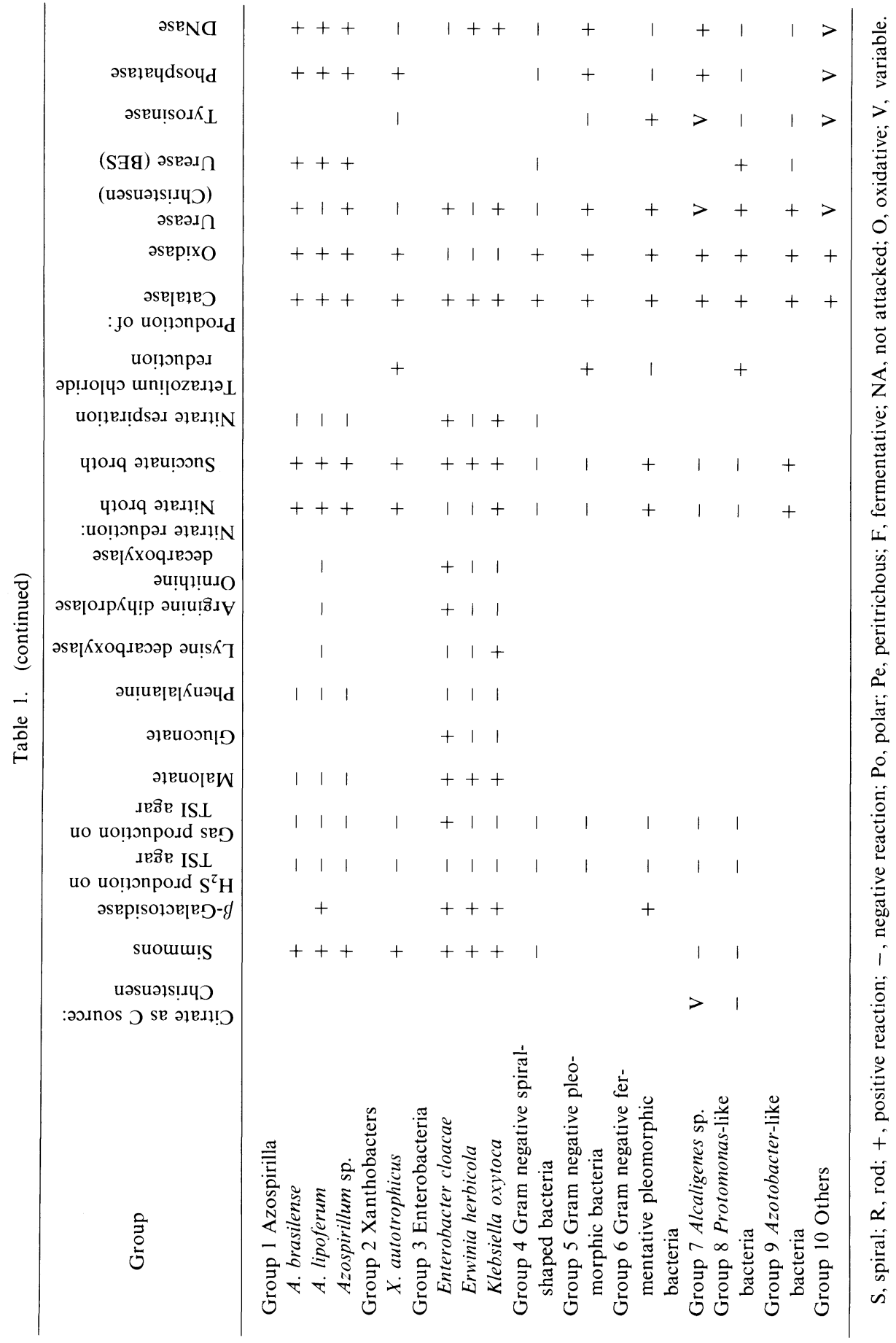


Table 2. Chemotaxonomic characters of

\begin{tabular}{|c|c|c|c|c|c|c|c|c|c|c|c|c|}
\hline \multirow{3}{*}{$\begin{array}{l}\text { Group of } \\
\text { strains }\end{array}$} & \multirow{3}{*}{$\begin{array}{c}\text { GC } \\
\text { content } \\
(\%)\end{array}$} & \multirow{3}{*}{$\begin{array}{l}\text { Quinone } \\
\text { system }\end{array}$} & \multicolumn{10}{|c|}{ Cellular } \\
\hline & & & \multicolumn{10}{|c|}{ Straight-chain acids } \\
\hline & & & $12: 0$ & $14: 0$ & $14: 1$ & $15: 0$ & $16: 0$ & $16: 1$ & $17: 0$ & $18: 0$ & $18: 1$ & $19: 0$ \\
\hline Group 1 SL-6 & 66.3 & Q-10 & $0.87 \mathrm{~b}$ & 0.79 & & 0.12 & 6.80 & 13.35 & 0.08 & 0.30 & 59.31 & \\
\hline $\operatorname{COC} 8$ & 66.8 & Q-10 & 1.03 & 0.70 & 1.66 & 1.94 & 5.46 & 17.34 & 0.12 & 0.32 & 44.14 & 0.37 \\
\hline SL-1 & 65.6 & Q-10 & 0.60 & 0.69 & 0.04 & 0.18 & 12.81 & 12.08 & & 0.36 & 60.78 & \\
\hline SL-2 & 65.5 & Q-10 & 0.58 & 0.66 & 0.05 & 0.14 & 8.25 & 14.01 & 0.31 & 0.34 & 61.66 & \\
\hline SL-9 & 67.5 & Q-10 & & & & & & & & & & \\
\hline SL-13 & 65.5 & Q-10 & 0.13 & 1.25 & 0.24 & 0.30 & 11.82 & 9.61 & 0.49 & 6.41 & 56.49 & \\
\hline SL-18 & 67.5 & Q-10 & & & & & & & & & & \\
\hline Group 2 Y-29 & 67.5 & Q-10 & 0.42 & 0.03 & & & 2.99 & 0.54 & 0.64 & 7.71 & 79.47 & \\
\hline $\mathrm{Y}-30$ & 64.3 & Q-10 & 1.15 & 0.17 & & 0.25 & 2.54 & 0.55 & & 4.44 & 77.33 & \\
\hline$Y-31$ & 63.1 & $\mathrm{Q}-10$ & & & & & 3.28 & 0.56 & 0.57 & 4.52 & 79.17 & \\
\hline$Y-32$ & 65.1 & Q-10 & 0.02 & 0.18 & $\mathrm{~T}$ & 0.34 & 3.89 & 0.48 & 0.74 & 3.52 & 70.66 & \\
\hline$Y-33$ & 64.3 & Q-10 & 0.20 & 0.07 & 0.04 & 0.02 & 2.98 & 0.99 & 0.52 & 4.62 & 74.11 & \\
\hline $\mathrm{Y}-34$ & 63.1 & Q-10 & 0.06 & 0.10 & & & 3.03 & 0.89 & 0.44 & 4.68 & 81.78 & \\
\hline$Y-35$ & 67.5 & Q-10 & & $\mathrm{T}$ & & & 3.41 & 0.64 & 0.64 & 4.47 & 77.71 & \\
\hline Y -36 & 66.3 & Q-10 & & & & & 3.33 & 0.67 & 0.69 & 4.74 & 79.22 & \\
\hline Group $3 \mathrm{Fe}-6$ & & Q-8 & 5.77 & 5.43 & 0.03 & 1.05 & 41.28 & 4.36 & 0.88 & 0.65 & 8.32 & \\
\hline $\mathrm{Fe}-3$ & & Q-8 & & & & & & & & & & \\
\hline $\mathrm{Fe}-4$ & & Q-8 & 6.72 & 7.77 & 0.06 & 1.53 & 27.25 & 9.80 & 0.74 & 0.39 & 15.62 & \\
\hline $\mathrm{Fe}-10$ & & Q-8 & 3.42 & 6.02 & 0.05 & 3.40 & 29.50 & 9.11 & 3.01 & 1.38 & 20.12 & \\
\hline NG 13 & & Q-8 & 5.32 & 6.55 & & 0.16 & 21.97 & 6.81 & $\mathrm{~T}$ & 0.36 & 511.44 & \\
\hline Group 4 SS-20 & 63.1 & Q-8 & 0.38 & 2.29 & & 0.18 & 34.06 & 47.91 & & 0.30 & 14.52 & \\
\hline SS-22 & 63.1 & Q-8 & 0.33 & 1.50 & 0.24 & 0.05 & 24.36 & 46.66 & & 0.56 & 25.88 & \\
\hline SS-25 & & Q-8 & 0.21 & 1.62 & 0.05 & & 32.72 & 47.70 & & 0.37 & 16.97 & \\
\hline SS- 28 & 65.1 & Q-8 & $\mathrm{T}$ & 2.77 & & & 36.65 & 46.45 & & 0.32 & 13.57 & \\
\hline SS-29 & 63.1 & Q-8 & 0.46 & 3.08 & 0.32 & 0.10 & 31.16 & 48.38 & & 0.29 & 15.89 & \\
\hline SS-32 & 65.3 & Q-8 & & & & & 32.36 & 47.70 & & 0.42 & 217.16 & \\
\hline Group 5 B-26 & 61.1 & Q-10 & 0.51 & 0.06 & 0.02 & 0.04 & 3.52 & 0.74 & 0.19 & 4.34 & 29.33 & \\
\hline B-45 & 61.6 & Q-10 & & & & & & & & & & \\
\hline B-40 & 61.9 & Q-10 & & & & & 3.26 & 0.83 & 0.25 & 7.21 & 59.16 & \\
\hline B-46 & 60.2 & Q-10 & & 0.04 & 0.04 & 0.08 & 1.44 & 0.56 & 0.05 & 3.57 & 62.76 & \\
\hline B-47 & 62.2 & Q-10 & & & & 0.06 & 2.58 & 1.22 & 0.09 & 2.32 & 45.62 & \\
\hline B-51 & 59.9 & Q-10 & 0.62 & 0.07 & 0.03 & 0.03 & 3.60 & 0.84 & 0.08 & 4.08 & 31.05 & \\
\hline Group 6 F-4 & 62.1 & Q-10 & & 0.32 & $\mathrm{~T}$ & 0.09 & 12.94 & 1.14 & 1.41 & 9.21 & 56.96 & \\
\hline F-7 & 63.1 & Q-10 & 0.39 & 0.38 & & 0.15 & 12.79 & 0.56 & 1.12 & 11.63 & 26.34 & \\
\hline B-2 & 62.8 & Q-10 & & 0.65 & 0.08 & 0.23 & 13.77 & 1.01 & 1.52 & 12.92 & 36.36 & \\
\hline B-4 & 62.2 & Q-10 & & 0.34 & 0.04 & 0.13 & 13.49 & 0.79 & 1.51 & 10.09 & 42.88 & \\
\hline B-7 & 62.6 & Q-10 & 0.40 & 0.30 & & 0.13 & 13.36 & 0.69 & 1.43 & 9.50 & 43.67 & \\
\hline B-18 & 62.9 & Q-10 & & 0.31 & & 0.16 & 10.44 & 1.31 & 1.34 & 5.90 & 60.64 & \\
\hline B-25 & 62.6 & Q-10 & & 0.44 & & 0.20 & 15.01 & 1.01 & 0.28 & 11.02 & 41.96 & \\
\hline B-32 & 63.1 & Q-10 & 0.04 & 0.54 & 0.99 & 0.16 & 13.03 & 0.91 & 1.13 & 8.55 & 34.87 & \\
\hline Group 7 OSG 47 & 66.3 & Q-10 & 0.88 & 1.72 & 5.47 & 0.30 & 18.78 & 1.33 & & 0.78 & 48.95 & \\
\hline$Y-22$ & 66.0 & Q-10 & 0.82 & 0.57 & $\mathrm{~T}$ & 0.26 & 17.58 & 0.48 & & 0.78 & 51.53 & \\
\hline$Y-23$ & 66.8 & Q-10 & 0.92 & 0.30 & 0.15 & 0.53 & 22.45 & 2.05 & & 0.45 & 46.16 & \\
\hline$Y-39$ & 68.0 & Q-10 & 0.89 & 0.31 & 0.19 & 0.08 & 22.65 & 1.09 & & 0.49 & 47.38 & \\
\hline Group 8 R-2 & 64.6 & Q-10 & 0.45 & 0.95 & & & 13.81 & 3.10 & 0.40 & 13.09 & 57.17 & 0.51 \\
\hline R-4 & 64.1 & Q-10 & & & & & & & & & & \\
\hline R-5 & 64.3 & Q-10 & $\mathrm{T}$ & 1.17 & & & 14.96 & 3.53 & 0.42 & 11.78 & 59.76 & 0.52 \\
\hline $\mathrm{R}-7$ & 65.1 & Q-10 & 0.39 & 0.73 & & & 12.66 & 2.57 & 0.24 & 13.62 & 257.99 & 0.30 \\
\hline
\end{tabular}


nitrogen-fixing bacteria and reference strains.

\begin{tabular}{|c|c|c|c|c|c|c|c|c|c|c|c|c|c|}
\hline \multicolumn{2}{|c|}{$\begin{array}{l}\text { Cyclopro- } \\
\text { pane acids }\end{array}$} & \multicolumn{7}{|c|}{ Hydroxy acids } & \multicolumn{5}{|c|}{ Unknown peaks } \\
\hline $17: 0$ & $19: 0$ & $\begin{array}{l}3-\mathrm{OH} \\
10: 0\end{array}$ & $\begin{array}{c}2-\mathrm{OH} \\
12: 0\end{array}$ & $\begin{array}{l}3-\mathrm{OH} \\
12: 0\end{array}$ & $\begin{array}{c}2-\mathrm{OH} \\
14: 0\end{array}$ & $\begin{array}{l}3-\mathrm{OH} \\
14: 0\end{array}$ & $\begin{array}{c}3-\mathrm{OH} \\
16: 0\end{array}$ & $\begin{array}{l}3-\mathrm{OH} \\
18: 0\end{array}$ & 1 & 2 & 3 & 4 & Others \\
\hline 1.08 & 0.86 & & & & & 6.30 & 3.36 & 1.97 & 1.77 & 1.29 & & & 1.74 \\
\hline 0.63 & 0.65 & & & & & 11.92 & 6.16 & 3.45 & 1.45 & 1.51 & & & 1.14 \\
\hline 0.60 & 0.63 & & & & & 5.66 & 3.07 & 1.61 & 1.52 & 1.28 & & & 1.28 \\
\hline 0.93 & 0.94 & & & & & 4.51 & 3.08 & 1.33 & 1.19 & 0.73 & & & 1.30 \\
\hline 0.76 & 0.26 & & & & & 3.59 & 2.25 & 0.42 & 0.71 & 3.31 & & & 2.22 \\
\hline 0.18 & 1.47 & & & & & & 1.76 & & 0.67 & & & & 4.77 \\
\hline 0.32 & 4.73 & & & & & & 4.16 & & & & & & 4.35 \\
\hline 0.21 & 4.03 & & & & & & 3.76 & & & & & & 3.90 \\
\hline 0.16 & 7.32 & & & & & & 5.70 & & 5.62 & & & & 1.66 \\
\hline 0.30 & 5.02 & & & & & & 4.03 & & 0.89 & & & & 6.18 \\
\hline 0.26 & 2.58 & & & & & & 2.93 & & 0.40 & & & & 2.40 \\
\hline 0.16 & 4.50 & & & & & & 4.18 & & $\mathrm{~T}$ & & & & 4.28 \\
\hline 0.22 & 4.19 & & & & & & 3.34 & & & & & & 3.63 \\
\hline 9.95 & 7.98 & & & & & 8.05 & & & & & & & 2.41 \\
\hline 6.81 & 7.77 & & & & & 13.81 & & & & & & & 1.04 \\
\hline 7.27 & 5.34 & & & & & 8.04 & & & & & & & 1.37 \\
\hline 2.25 & 8.50 & & & & & 32.42 & & & & & & & 4.02 \\
\hline 0.10 & & & & & 0.26 & & & & & & & & \\
\hline 0.07 & & & & & 0.27 & & & & & & & & \\
\hline \multirow[t]{4}{*}{0.03} & & & & & 0.23 & & & & & & & & \\
\hline & & & & & 0.25 & & & & & & & & \\
\hline & & & & & 0.26 & & & & & & & & \\
\hline & & & & & 0.24 & & & & & & & & \\
\hline 0.06 & 18.91 & & & & & 4.59 & 4.67 & 5.38 & & & 14.63 & & 13.01 \\
\hline 0.03 & 4.48 & & & & & 5.03 & 1.92 & 5.48 & & & 6.28 & & 6.07 \\
\hline 0.45 & 5.04 & & & & & 4.49 & 3.19 & 4.48 & & & 5.27 & & 8.54 \\
\hline 0.67 & 5.06 & & & & & 11.71 & 4.02 & 11.75 & & & 10.97 & & 3.93 \\
\hline 0.08 & 17.35 & & & & & 3.86 & 4.56 & 6.16 & & & 14.60 & & 12.99 \\
\hline 0.15 & 5.17 & & & & & 3.43 & 1.09 & 0.77 & & & 4.52 & & 2.80 \\
\hline 0.32 & 15.53 & & & & & 2.83 & 3.62 & 1.38 & & & 12.57 & & 10.39 \\
\hline 0.28 & 9.70 & & & & & 3.05 & 2.56 & 1.28 & & & 9.09 & & 7.50 \\
\hline 0.88 & 8.50 & & & & & 3.49 & 2.40 & 1.18 & & & 8.50 & & 6.08 \\
\hline 0.24 & 9.50 & & & & & 3.76 & 2.22 & 1.20 & & & 8.12 & & 5.48 \\
\hline 0.29 & 5.51 & & & & & 3.91 & 4.94 & 1.11 & & & 0.17 & & 3.97 \\
\hline 0.28 & 8.34 & & & & & 2.35 & 2.08 & 0.76 & & & 8.54 & & 7.73 \\
\hline 0.31 & 10.22 & & & & & 6.27 & 3.24 & 2.53 & & & 10.66 & & 6.55 \\
\hline 0.57 & 0.52 & & & & 8.36 & & & & & & & 8.90 & 3.44 \\
\hline 0.69 & 1.12 & & & & 14.43 & & & & & & & 11.11 & \\
\hline 0.28 & 0.31 & & & & 16.05 & & & & & & & 8.65 & 1.70 \\
\hline 0.34 & 0.34 & & & & 16.16 & & & & & & & 9.22 & \\
\hline 0.28 & & & & & & 2.20 & & & & & & 6.66 & 1.38 \\
\hline 0.24 & & & & & & 3.96 & & & & & & 2.20 & 1.46 \\
\hline 0.24 & & & & & & 2.11 & & & & & & 8.16 & 0.99 \\
\hline
\end{tabular}


Table 2.

\begin{tabular}{ccccc} 
& & & Cellular \\
\cline { 3 - 4 } $\begin{array}{c}\text { Group of } \\
\text { strains }\end{array}$ & $\begin{array}{c}\text { GC } \\
\text { content } \\
(\%)\end{array}$ & $\begin{array}{c}\text { Quinone } \\
\text { system }\end{array}$ & Straight-chain acids & \\
\cline { 4 - 5 } & & &
\end{tabular}

$12: 0 \quad 14: 014: 115: 0 \quad 16: 0 \quad 16: 1 \quad 17: 0 \quad 18: 0 \quad 18: 1 \quad 19: 0$

\begin{tabular}{|c|c|c|c|c|c|c|c|c|c|c|c|c|}
\hline Group 8 R-11 & 64.1 & Q-10 & 0.06 & 0.99 & & & 15.01 & 3.33 & 0.41 & 13.35 & 56.41 & 0.46 \\
\hline $\mathrm{R}-12$ & 64.3 & Q-10 & 0.28 & 1.08 & & & 15.61 & 3.22 & 0.46 & 14.45 & 57.82 & 0.36 \\
\hline $\mathrm{R}-13$ & 63.6 & Q-10 & 0.17 & 0.76 & & & 13.43 & 2.22 & 0.23 & 13.83 & 60.63 & \\
\hline $\mathrm{R}-15$ & 62.6 & Q-10 & 0.27 & 0.95 & & & 13.91 & 3.17 & 0.40 & 11.22 & 56.56 & 0.52 \\
\hline Group 9 A-2 & 64.1 & Q-8 & 2.14 & 0.71 & 0.60 & 0.31 & 29.28 & 54.98 & & 1.24 & 7.27 & \\
\hline A-3 & 65.1 & Q-8 & 1.92 & 0.92 & 0.37 & 0.49 & 27.89 & 54.13 & & 2.56 & 8.70 & \\
\hline A-7 & 65.1 & Q-8 & 1.82 & 0.48 & 0.25 & & 26.22 & 58.00 & & $\mathrm{~T}$ & 9.26 & \\
\hline A-24 & & Q-8 & 2.34 & 0.58 & 0.22 & 0.22 & 24.09 & 58.04 & & 1.31 & 8.40 & \\
\hline \multicolumn{13}{|c|}{ Agrobacterium radiobacter } \\
\hline $\begin{array}{l}\text { IAM } 12048^{\mathrm{T}} \\
\text { A. tumefaciens }\end{array}$ & 59.2 & Q-10 & 0.02 & 1.50 & 0.22 & 0.21 & 30.33 & 0.11 & 0.04 & 0.50 & 8.49 & 3.18 \\
\hline IAM 1037 & 59.4 & Q-10 & & 0.50 & 0.06 & 0.44 & 30.65 & & 0.09 & 0.54 & 5.32 & 1.69 \\
\hline \multicolumn{13}{|c|}{ Alcaligenes denitrificans } \\
\hline $\begin{array}{l}\text { IAM } 12370 \\
\text { A. eutrophus }\end{array}$ & 67.5 & Q-8 & & & & 0.18 & 34.23 & & 0.76 & 1.45 & & 14.22 \\
\hline $\begin{array}{l}\text { IAM } 12368^{\mathrm{T}} \\
\text { A. faecalis }\end{array}$ & 65.8 & Q-8 & 0.20 & 7.14 & & 1.35 & 38.15 & 22.44 & & 0.44 & 6.75 & 2.09 \\
\hline $\begin{array}{l}\text { IAM } 12369 \\
\text { A. latus }\end{array}$ & 57.2 & Q-8 & 0.04 & 1.64 & & 0.68 & 36.21 & 3.87 & 0.86 & 0.95 & 12.80 & 9.79 \\
\hline $\begin{array}{l}\text { IAM } 12599 \\
\text { A. paradoxus }\end{array}$ & 69.2 & Q-8 & 1.70 & 3.68 & 0.05 & 0.08 & 22.86 & 40.33 & 0.09 & 5.96 & 24.85 & \\
\hline $\begin{array}{c}\text { DSM } 30034 \\
\text { Azomonas agilis }\end{array}$ & 68.0 & Q-8 & 3.11 & 1.50 & & 9.81 & 25.82 & 7.27 & 6.13 & 0.54 & 8.56 & 0.23 \\
\hline $\begin{array}{c}\text { DSM } 375 \\
\text { Azospirillum bra }\end{array}$ & & Q-9 & & 3.12 & & & 67.72 & 12.48 & & & 6.52 & \\
\hline $\begin{array}{l}\text { IAM } 12400 \\
\text { A. lipoferum }\end{array}$ & 69.0 & Q-10 & 1.96 & 0.98 & 0.11 & 0.10 & 3.73 & 14.59 & & $\mathrm{~T}$ & 40.21 & \\
\hline $\begin{array}{l}\text { IAM } 12399^{\mathrm{T}} \\
\text { Azotobacter vine }\end{array}$ & 69.0 & Q-10 & 0.88 & 0.52 & 1.00 & 1.02 & 6.40 & 9.11 & & 0.35 & 49.60 & 0.18 \\
\hline $\begin{array}{l}\text { DSM } 87 \\
\text { Beijerinckia indi }\end{array}$ & & Q-8 & & 3.48 & 0.14 & $\mathrm{~T}$ & 41.48 & $28: 97$ & 0.82 & & 8.88 & \\
\hline $\begin{array}{c}\text { MCIB } 8712 \\
\text { Derxia gummoss }\end{array}$ & & Q-10 & & & & & & & & & & \\
\hline $\begin{array}{c}\text { NCIB } 9064 \\
\text { Klebsiella oxyto }\end{array}$ & 69.2 & Q-8 & 4.57 & 0.52 & & 0.07 & 15.37 & 31.76 & & $\mathrm{~T}$ & 33.74 & \\
\hline $\begin{array}{l}\text { RH } 1586 \\
\text { Protomonas ext }\end{array}$ & & Q-8 & 4.08 & 5.21 & 8.24 & 0.10 & 27.92 & 11.33 & $\mathrm{~T}$ & 1.03 & 13.98 & \\
\hline TK 0001 & 65.6 & Q-10 & & & & & $4.3 \mathrm{a}$ & 13.1 & & 2.1 & 72.7 & \\
\hline Pseudomonas pa & bilis & & & & & & & & & & & \\
\hline $\mathrm{KM} 2395^{\mathrm{T}}$ & 64.3 & Q-10 & & 9.90 & & & 16.35 & 2.09 & & $\mathrm{~T}$ & 65.68 & \\
\hline KM 2397 & 65.8 & $\mathrm{Q}-10$ & 0.33 & 4.97 & & & 9.27 & 6.99 & & 0.49 & 58.76 & \\
\hline Xanthobacter au & hicus & & & & & & & & & & & \\
\hline DSM 431 & 67.2 & $Q-10$ & & 0.25 & & & 4.70 & 4.56 & 0.14 & 4.28 & 79.24 & \\
\hline $\operatorname{DSM} 432^{\mathrm{T}}$ & 67.0 & Q-10 & & 0.24 & & & 4.93 & 4.81 & 0.13 & 3.83 & 80.52 & \\
\hline$X$. flavus & & & & & & & & & & & & \\
\hline NCIB 10071 & & Q-10 & 0.06 & 1.19 & & & 3.19 & 0.25 & 0.17 & 5.58 & 85.59 & \\
\hline
\end{tabular}

Symbols: a, This value was obtained from ref. 63 ; $\mathrm{b}$, Numbers are percentages of total acids; $\mathrm{T}$, acid 
(continued)

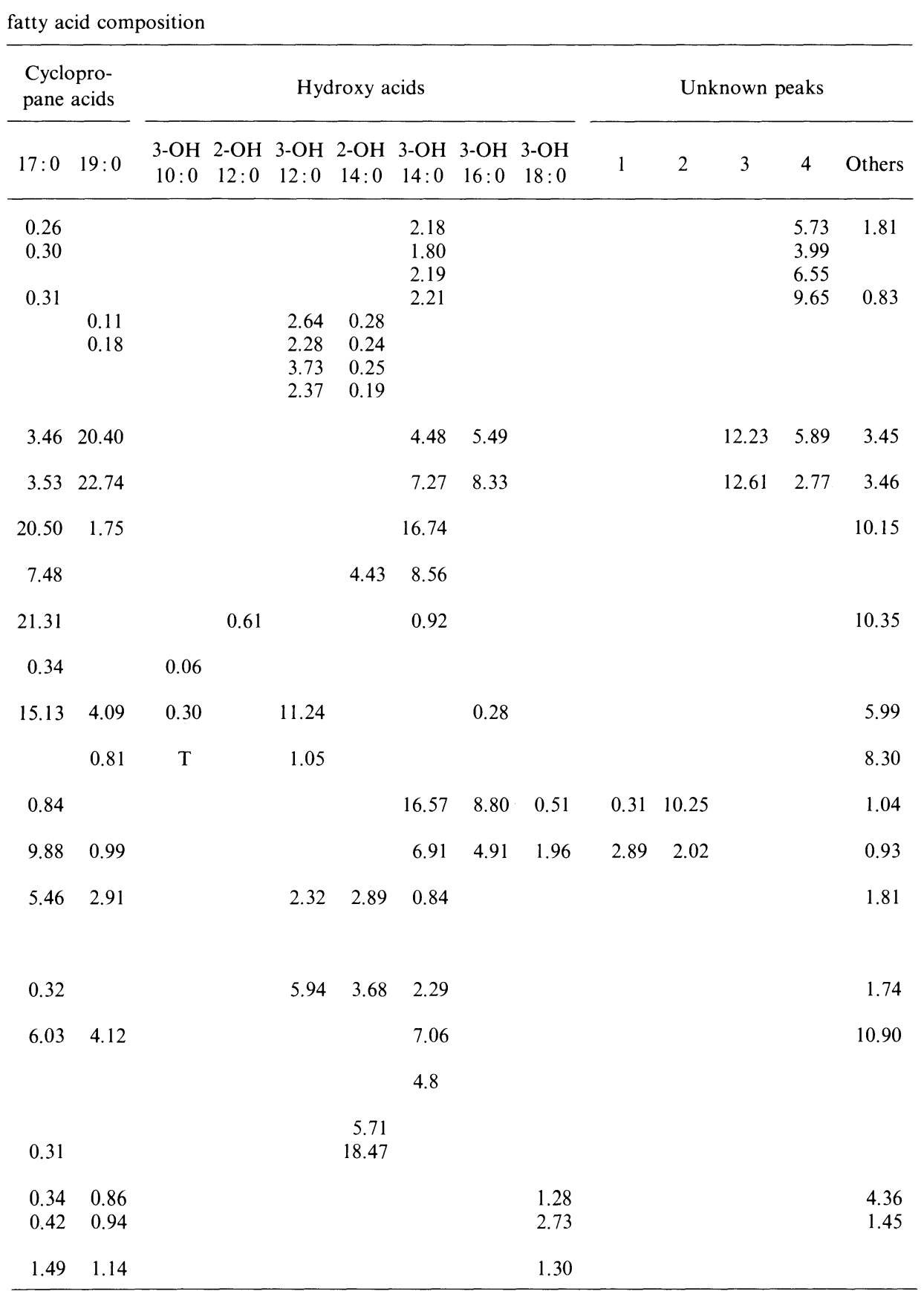

present at less than $0.01 \%$. 
Table 3. Production of acid from carbon compounds by strains of group 1 (Azospirilla) and reference strains

\begin{tabular}{|c|c|c|c|c|c|c|c|c|c|}
\hline \multirow{2}{*}{$\begin{array}{l}\text { Carbon } \\
\text { compound }\end{array}$} & \multicolumn{9}{|c|}{ Strain } \\
\hline & SL-6 & $\operatorname{COC} 8$ & SL-1 & SL-2 & SL-9 & SL-13 & SL-18 & A. brasilense $e^{a}$ & A. lipoferum ${ }^{\circ}$ \\
\hline L-Arabinose & - & + & - & + & + & - & + & $\mathrm{V}$ & + \\
\hline D-Xylose & - & + & + & + & + & + & + & - & V \\
\hline D-Ribose & + & + & + & + & + & + & + & + & + \\
\hline D-Glucose & - & + & + & + & + & + & + & V & + \\
\hline D-Fructose & + & + & + & + & + & + & + & + & + \\
\hline D-Galactose & - & + & + & + & + & + & + & $\mathrm{V}$ & + \\
\hline Lactose & - & - & - & - & - & - & - & - & - \\
\hline Maltose & - & - & - & - & - & - & - & - & - \\
\hline L-Rhamnose & - & + & - & - & - & - & - & - & - \\
\hline Cellobiose & - & - & + & + & - & - & - & - & - \\
\hline Sorbitol & - & - & - & - & - & - & - & V & - \\
\hline Mannitol & - & - & + & - & + & + & - & - & $\mathrm{V}$ \\
\hline Inositol & - & - & + & + & - & + & + & - & V \\
\hline
\end{tabular}

Symbols: + , positive reaction; - , negative reaction; $\mathrm{V}$, variable.

a These data were taken from Bergey's Manual of Systematic Bacteriology, Vol. 1 (5l).

SL-6 had $66.3 \mathrm{~mol} \% \mathrm{G}+\mathrm{C}$ and the ubiquinone system (Q-10) as shown in Table 2. Straight-chain unsaturated $\mathrm{C}_{18: 1}$ fatty acid was the major fatty acid, and 3-hydroxy $\mathrm{C}_{14: 0}\left(3-\mathrm{OH} \mathrm{C} \mathrm{C}_{14: 0}\right), 3-\mathrm{OH} \mathrm{C} \mathrm{C}_{16: 0}$, and 3-OH C $\mathrm{C}_{18: 0}$ were also found.

On the basis of the above characters the strain SL-6 belongs to the genus Azospirillum. This genus includes 4 species, $A$. amazonense, $A$. brasilense, $A$. halopraeferens, and $A$. lipoferum $(29,49,50)$. Azospirillum lipoferum differed from $A$. brasilense in the production of acid from glucose and mannitol, utilization of glucose as a sole carbon source for growth in nitrogen-free semisolid medium, requirement of biotin, and DNA-DNA hybridization (29), from $A$. amazonense in GC content, cell size, and the presence of the pili (49), and from $A$. halopraeferens in the tolerance of $\mathrm{NaCl}(50)$. Chemotaxonomic characters of $A$. lipoferum IAM $12399^{\mathrm{T}}$ and $A$. brasilense IAM $12400^{\mathrm{T}}$ are also shown in Table 2. Azospirillum lipoferum could not be differentiated from $A$. brasilense in the chemotaxonomic characters of the GC content, fatty acid composition, and quinone system.

Strain SL-6 was identified as A. brasilense Tarrand, Krieg and Döbereiner 1979 because it did not produce acid from glucose, did not utilize glucose as a sole carbon source, and did not require biotin.

Source: Strain SL-6 was isolated in 1982 from the root of Oryza sativa strain C5444.

Azospirillum lipoferum Tarrand, Krieg and Döbereiner 1979

Strain: COC 8

The morphological characters of strain COC 8 are similar to those of strain SL- 


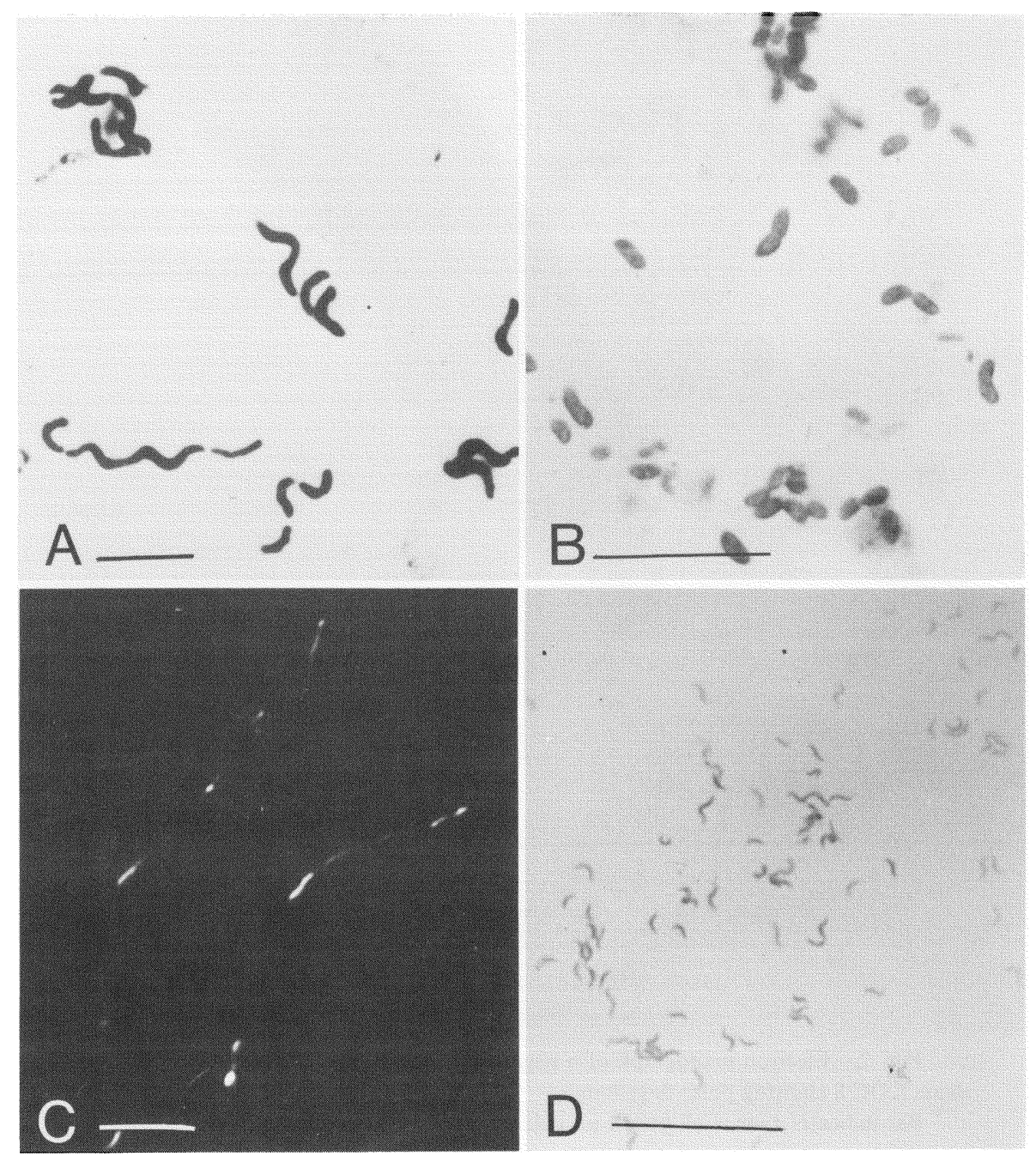

Fig. 1. Photomicrographs of strains of groups 1 and 4 .

A: Azospirillum sp. strain SL-1 (group 1) grown on M semisolid agar medium for 1 day at $30^{\circ} \mathrm{C}$ (optical microscopy, stained with crystal violet). B: Azospirillum lipoferum strain $\mathrm{COC} 8$ (group 1) grown on nutrient agar medium for 1 day at $30^{\circ} \mathrm{C}$ (optical microscopy, stained with crystal violet). C: PHB granules in Azospirillum lipoferum strain COC 8 stained with Nile blue A and observed under fluorescent light. D: Gram negative spiral-shaped bacterial strain SS-22 (group 4) grown on nutrient agar medium for 1 day at $30^{\circ} \mathrm{C}$ (optical microscopy, stained with crystal violet). Bars indicate $10 \mu \mathrm{m}$.

6. The strain formed spiral or vibrio-like cells as shown in Figs. 1B, 1C, and 2. It had a polar flagellum as shown in Fig. 2 .

The strain produced acid from glucose aerobically and anaerobically, and used 


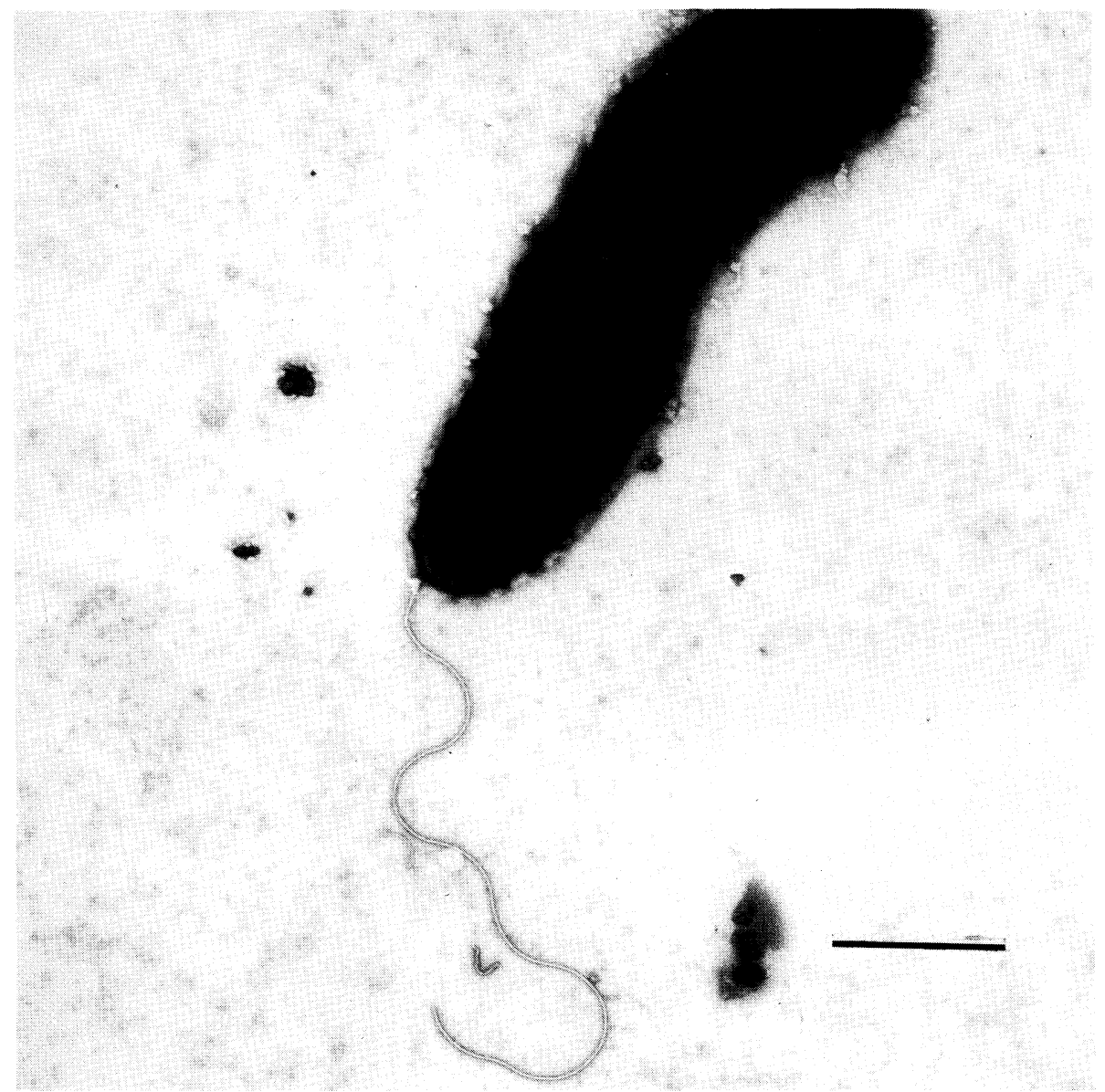

Fig. 2. Electron micrograph of a negatively stained cell of Azospirillum lipoferum strain COC 8 showing polar flagellum.

Bar indicates $1 \mu \mathrm{m}$. Cell grown on nutrient medium for 1 day at $30^{\circ} \mathrm{C}$.

glucose as a sole carbon source by the method of TARRAND et al. (29). Strain COC 8 produced acid from L-arabinose, D-xylose, D-ribose, D-glucose, D-fructose, Dgalactose, and L-rhamnose but not from sorbitol, lactose, maltose, mannitol, inositol, cellobiose, and sucrose. The strain required biotin. It utilized hydrogen.

Strain COC 8 had $66.8 \mathrm{~mol} \% \mathrm{G}+\mathrm{C}$ and a ubiquinone system (Q-10) as shown in Table 2. The fatty acid composition was similar to those of strains SL-6, $A$. brasilense IAM $12400^{\mathrm{T}}$, and $A$. lipoferum IAM $12399^{\mathrm{T}}$.

Consequently strain COC 8 was identified as A. lipoferum Tarrand, Krieg and Döbereiner 1979 from the characters mentioned above.

Source: Strain COC 8 was isolated in 1980 from the roots of Oryza sativa strain C5444. 
Azospirillum sp.

Strains: SL-1, SL-2, SL-9, SL-13, and SL-18

The morphological and biochemical characters of these strains were similar to those of strain COC 8. The cell shape of strain SL-1 is shown in Fig. 1A. The production of acid from L-arabinose, D-xylose, D-glucose, D-galactose, L-rhamnose, cellobiose, mannitol, and inositol differed among strains as shown in Table 3. The utilization of hydrogen was investigated in strains SL-1, SL-2, and SL-18. These three strains utilized hydrogen.

The chemotaxonomic characters of isolates are shown in Table 2. The GC content of the strains ranged from 65.5 to $67.5 \%$. The fatty acid composition was similar to that of strain COC 8, and the quinone system was Q-10.

The strains studied belong to the genus Azospirillum. They utilized glucose as a sole carbon source for growth and produced acid from glucose and fructose aerobically and anaerobically, although they did not require biotin. From these characters, these strains were not identified as belonging to a known species in the genus Azospirillum.

Source: Strains SL-1, SL-2 and SL-13 were isolated in 1982 from the roots of Oryza sativa strain C5444. Strain SL-18 was isolated in 1981 from the roots of Oryza sativa strain C5444. Strain SL-9 was isolated in 1982 from the roots of Oryza sativa strain T65.

Group 2. Xanthobacters

Xanthobacter autotrophicus (Baumgarten, Reh and Schlegel 1974) Wiegel, Wilke, Baumgarten, Opitz and Schlegel 1978

Strains: Y-29, Y-30, Y-31, Y-32, Y-33, Y-34, Y-35, and Y-36

These strains had non-motile, pleomorphic, gram negative cells as shown in Fig. 3. The cells were 0.5 to $1.0 \mu \mathrm{m}$ by 1.0 to $5.0 \mu \mathrm{m}$. PHB granules were accumulated in the cells. Colonies were yellow due to a water-insoluble pigment. The strains did not produce acid from glucose oxidatively or fermentatively. They did not grow on Tellurite agar medium. Nor did they hydrolyze gelatin or starch. They reduced nitrate and tetrazolium chloride, and produced catalase, oxidase, and phosphatase. They did not produce urease, tyrosinase, or DNase. Utilization of hydrogen was investigated for strains Y-29, Y-31, and Y-32. These strains grew at the expense of hydrogen. Table 4 shows the results of assimilation of 55 carbon compounds. Assimilation of L-arabinose, D-xylose, D-ribose, maltose, mannose, glyoxylate, $\beta$-alanine, and L-valine differed among the strains as shown in Table 4.

The GC content of the strains studied ranged from 63.1 to $67.5 \%$ as shown in Table 2. They had the ubiquinone system Q-10. The isolates contained a large amount of straight-chain unsaturated fatty acid of $\mathrm{C}_{18: 1}(67-82 \%)$, and only a small amount of 3-OH $\mathrm{C}_{16: 0}$ acid.

From these characters the isolates apparently belong to the genus Xanthobacter. The genus Xanthobacter includes two species, $X$. autotrophicus and $X$. flavus. Xanthobacter autotrophicus differs from $X$. flavus in requiring the growth 

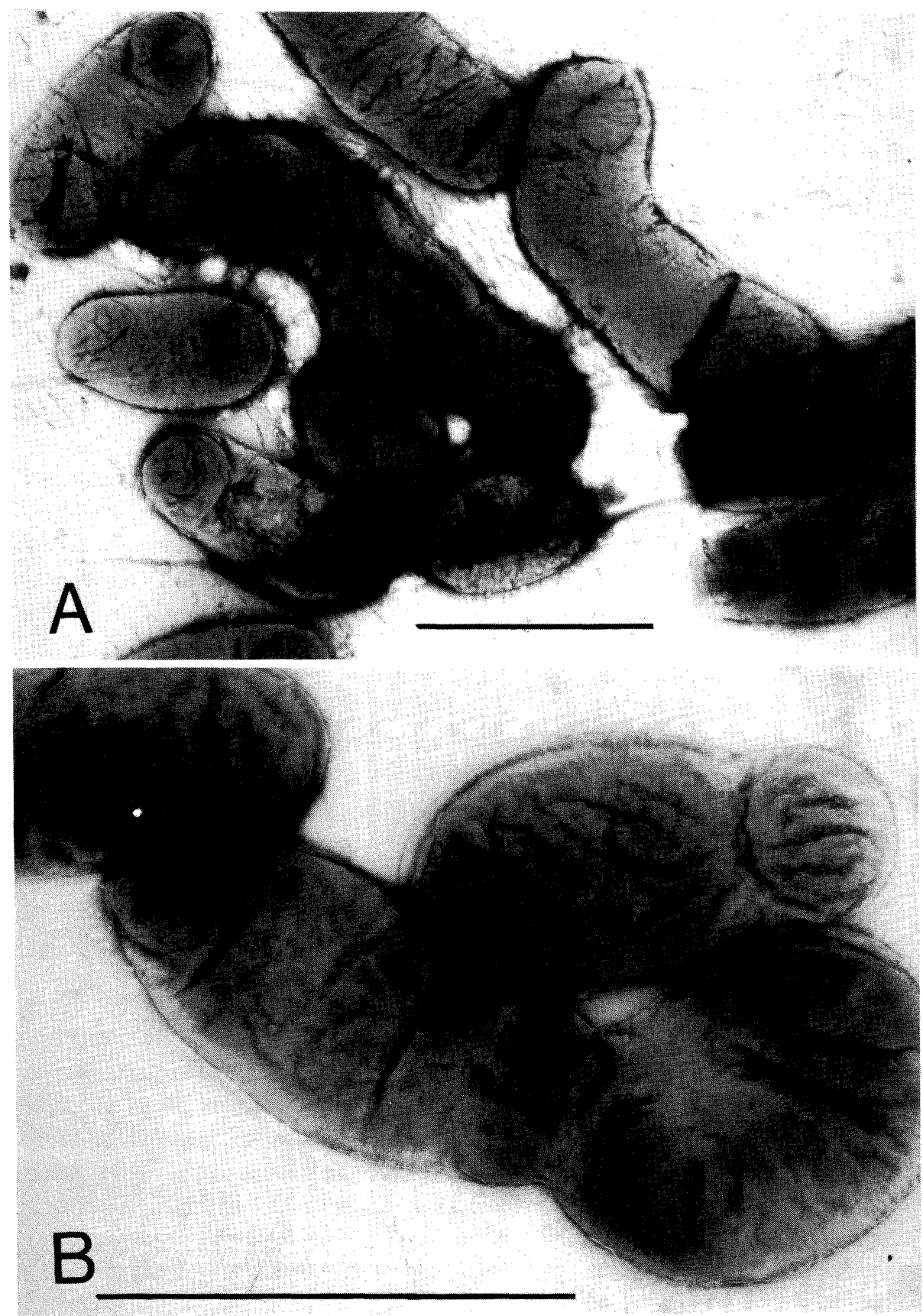

Fig. 3. Electron micrographs of Xanthobacter autotrophicus strains of group 2.

A: strain Y-30. B: strain Y-34. These cells were grown on nutrient agar medium for 1 day at $30^{\circ} \mathrm{C}$. Bars indicate $1 \mu \mathrm{m}$. 


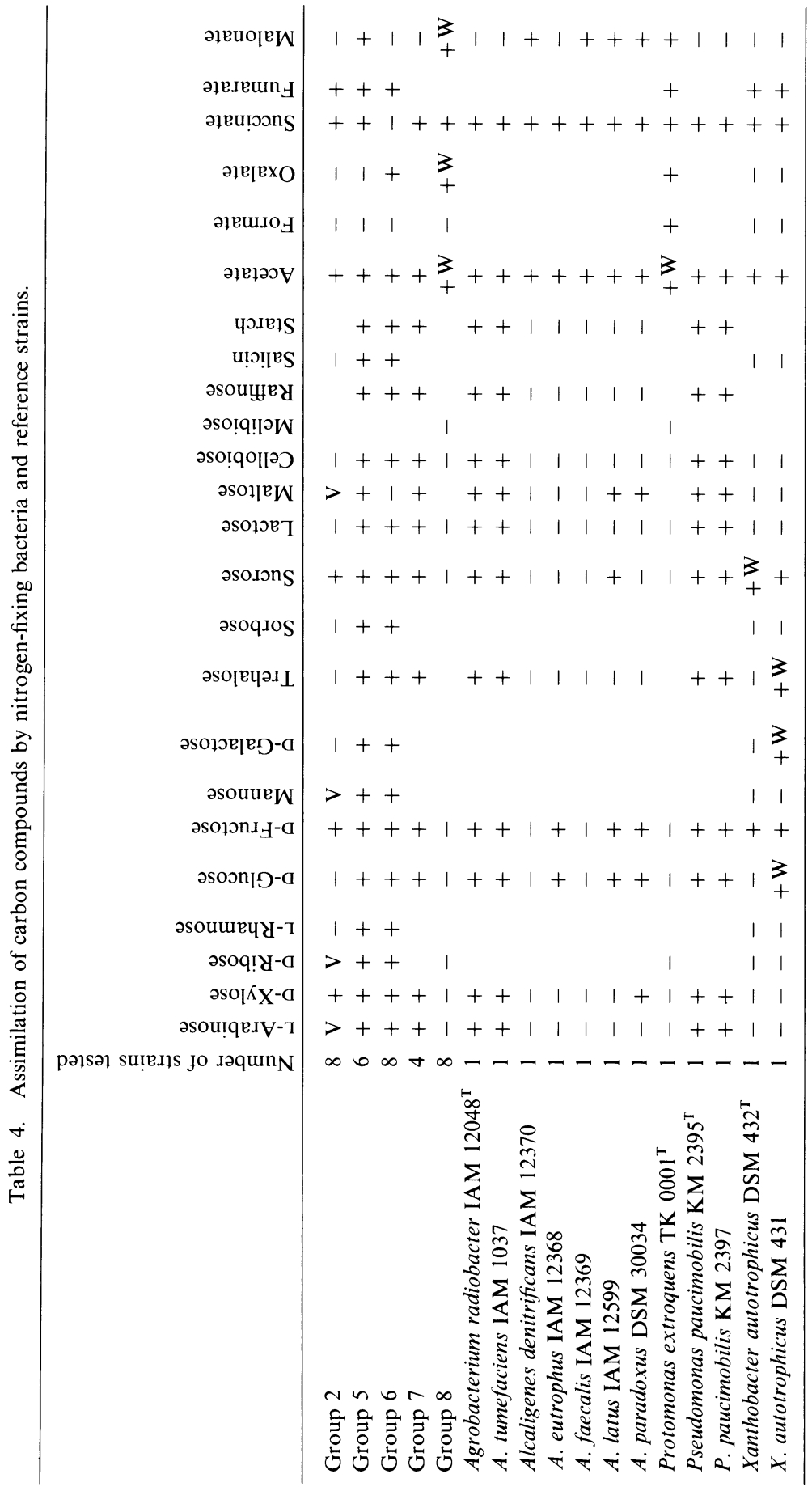




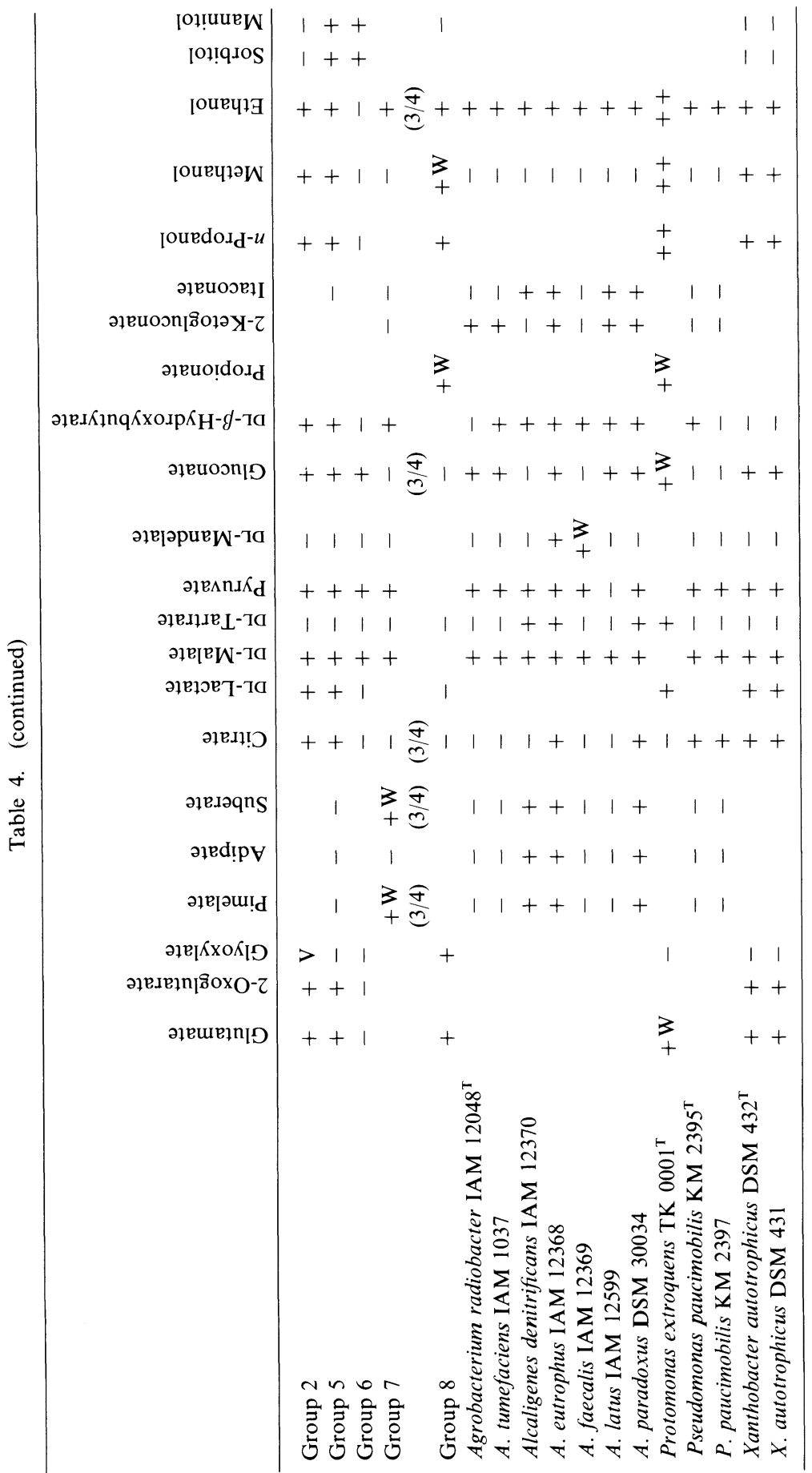




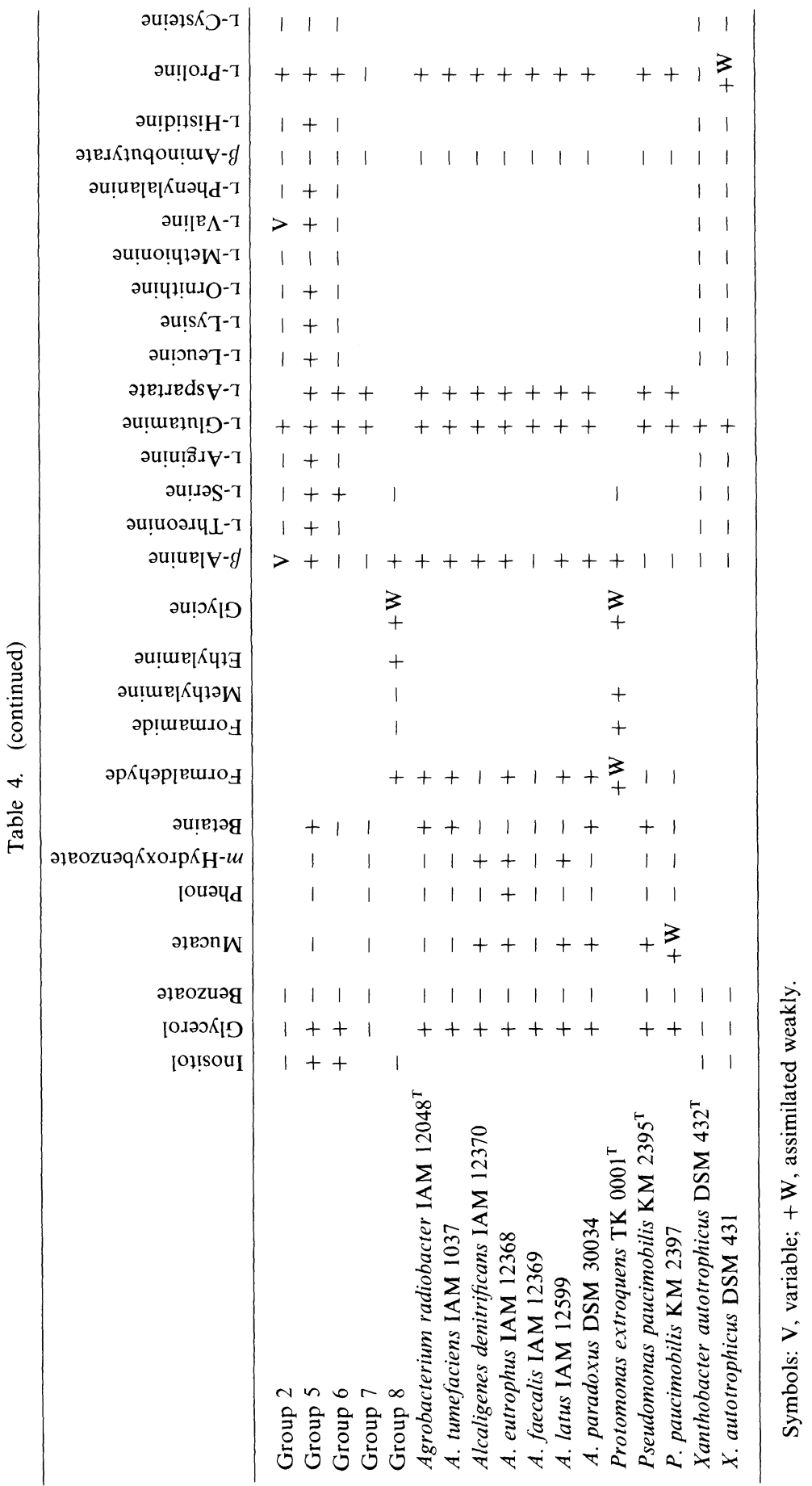


Table 5. Assimilation of carbon compounds by strains of group 2 (Xanthobacters) and $X$. autotrophicus (DSM 431 and DSM $432^{\mathrm{T}}$ ).

\begin{tabular}{lccccccccccc}
\hline \multicolumn{1}{c}{ Carbon compound } & Y-29 & Y-30 & Y-31 & Y-32 & Y-33 & Y-34 & Y-35 & Y-36 & $\begin{array}{c}\text { DSM } \\
431\end{array}$ & $\begin{array}{c}\text { DSM } \\
432^{\mathrm{T}}\end{array}$ \\
\hline L-Arabinose & - & + & - & + & + & - & + & - & - & - \\
D-Xylose & + & + & + & + & + & + & + & + & - & - \\
D-Ribose & - & + & - & + & + & - & + & - & - & - \\
D-Glucose & - & - & - & - & - & - & - & - & - & + W \\
D-Galactose & - & - & - & - & - & - & - & - & - & + W \\
Trehalose & - & - & - & - & - & - & - & - & - & + W \\
Maltose & + & + & + & - & + & - & - & - & - & - \\
Mannose & + & - & - & - & - & - & - & - & - & - \\
DL- $\beta$-Hydroxybutylate & + & + & + & + & + & + & + & + & - & - \\
Glyoxylate & - & - & - & - & - & - & + & - & - & - \\
$\beta$-Alanine & + & + & + & + & - & + & - & - & - & - \\
L-Valine & - & + & - & - & + & - & - & - & - & - \\
\hline
\end{tabular}

Symbols: + , assimilated; $+\mathrm{W}$, assimilated weakly; - , not assimilated.

factor. Like $X$. autotrophicus DSM 431 and DSM $432^{\mathrm{T}}$, the strains studied did not require the growth factor. Moreover the strains of this group resemble $X$. autotrophicus. The strains produced catalase, oxidase, and phosphatase and did not produce DNase, urease, tyrosinase. The strains of this group and the $X$. autotrophicus reference strains (DSM 431 and DSM $432^{\mathrm{T}}$ ) assimilated D-fructose, sucrose, acetate, succinate, fumarate, glutamate, 2-oxo-glutarate, citrate, DL-lactate, DL-malate, pyruvate, gluconate, $n$-propanol, methanol, ethanol, L-glutamine, and Lproline, but did not assimilate L-rhamnose, sorbose, lactose, cellobiose, salicin, formate, oxalate, malonate, DL-tartrate, DL-mandelate, sorbitol, mannitol, inositol, glycerol, benzoate, L-threonine, L-serine, L-arginine, L-leucine, L-lysine, L-ornithine, L-methionine, L-phenylalanine, $\beta$-aminobutyrate, L-histidine, and L-cysteine. However, the strains of this group assimilated DL- $\beta$-hydroxybutyrate but $X$. autotrophicus DSM 431 and $432^{\mathrm{T}}$ did not. The strains of this group and $X$. autotrophicus DSM 431 did not assimilate D-glucose, D-galactose, and trehalose but $X$. autotrophicus DSM $432^{\mathrm{T}}$ did so weakly.

Consequently, strains of group 2 were identified as $X$. autotrophicus (Baumgarten, Reh and Schlegel 1974) Wiegel, Wilke, Baumgarten, Opitz and Schlegel 1978.

This is the first report of the isolation of $X$. autotrophicus from the roots of rice.

Source: Strains Y-29, Y-30, and Y-31 were isolated in 1982 from the roots of Oryza sativa strain C5444. Strains Y-32, Y-33, Y-34, Y-35, and Y-36 were isolated in 1981 from the roots of Oryza sativa strain T65.

Group 3. Enterobacteria

Enterobacter cloacae (Jordon 1890) Hormaeche and Edwards 1960

Strains: OO 13a, X-1, X-7, and Fe-6 


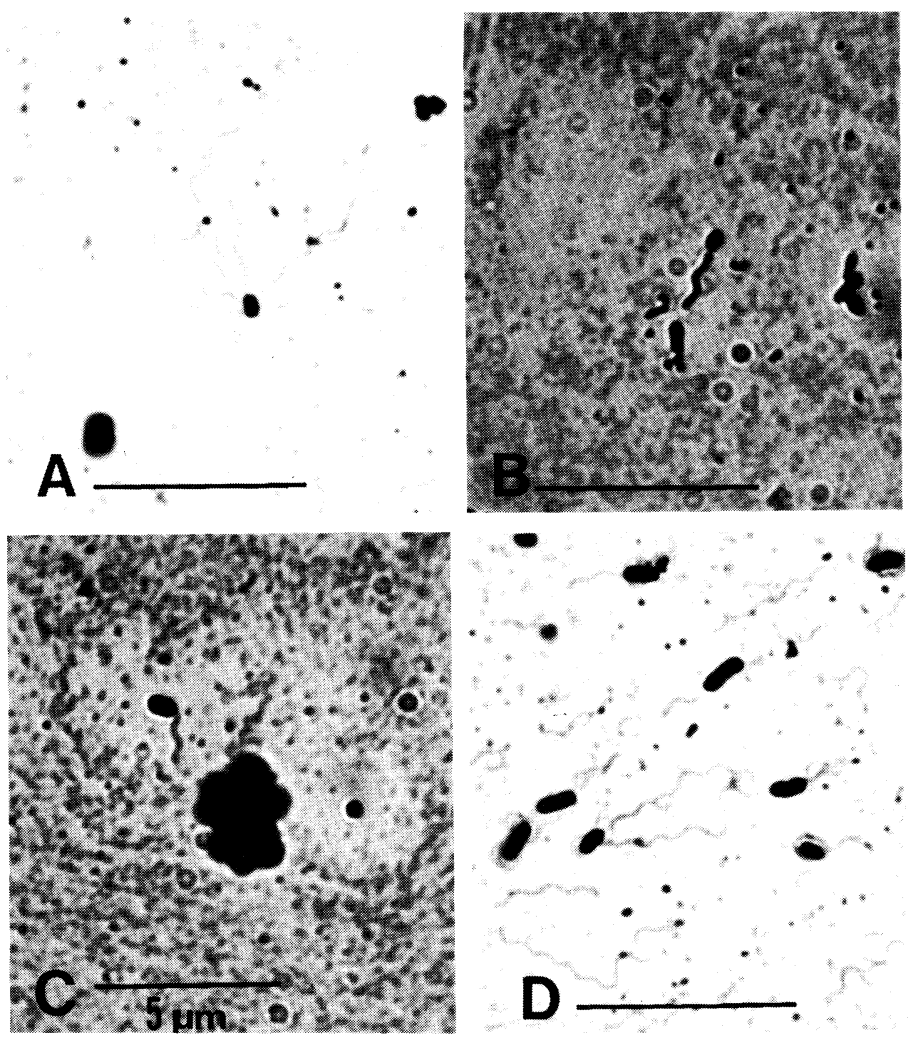

Fig. 4. Photomicrographs of nitrogen-fixing bacteria grown on nutrient agar medium for 1 day at $30^{\circ} \mathrm{C}$.

A: Enterobacter cloacae strain OO 13a (group 3). B: Protomonas-like bacteria strain R-5 (group 8). C: Azotobacter-like bacteria strain A-2 (group 9). D: Alcaligenes sp. strain OSG 47 (group 7). Bars indicate $10 \mu \mathrm{m}$.

Strains OO 13a, X-1, X-7, and Fe-6 were fermentative gram negative rods, and were motile with peritrichous flagella as shown in Fig. 4A. They formed circular, smooth, convex, and entire colonies on nutrient agar medium. Colonies on nutrient agar plate were light brown. The morphological and phenotypic characters of the strains studied are shown in Tables 1 and 6. Strains OO 13a, X-1, X-7, and Fe-6 liquefied gelatin and esculin. They showed positive reactions for VP-reaction, nitrate respiration, production of gas on TSI agar, $\beta$-galactosidase, catalase, arginine dihydrolase, ornithine decarboxylase, and oxidation of gluconate. They did not produce the pigment on gluconate medium, indole, hydrogen sulfide, phenylalanine deaminase, oxidase, urease, and DNase. They did not decarboxylate lysine. Strains OO 13a, X-1, and X-7 did not produce acid from L-arabinose but strain Fe-6 did.

Strain Fe-6 contained a large amount of straight-chain $\mathrm{C}_{16: 0}, \mathrm{C}_{18: 0}$, and 3-OH 
$\mathrm{C}_{14: 0}$ acids. The fatty acid composition of strain Fe-6 was similar to that of Klebsiella oxytoca RH 1586 as shown in Table 2. Strain Fe-6 had the ubiquinone system Q-8.

The strains differed from Enterobacter cloacae IAM 12349 in the assimilation of polygalacturonate and hydrolysis of gelatin at $20^{\circ} \mathrm{C}$. However, the strains were identified as Enterobacter cloacae (Jordon 1890) Hormaech and Edwards 1960 on the basis of motility with peritrichous flagella, and they showed oxidation of gluconate, production of arginine dihydrolase and ornithine decarboxylase, and liquefaction of gelatin at $30^{\circ} \mathrm{C}$. They did not produce lysine decarboxylase, urease, indole, and the pigment from gluconate medium, and did not assimilate inulin. They did not produce acid from dulcitol, adonitol, and inulin.

Source: Strains OO 13a, X-1, and X-7 were isolated from paddy soil. Strain Fe-6 was isolated in 1981 from the roots of Oryza sativa strain T65.

Erwinia herbicola (Löhnis 1911) Dye 1964

Strains: $\mathrm{Fe}-3$ and $\mathrm{Fe}-4$

The strains studied were fermentative gram negative rods, motile with peritrichous flagella. They formed smooth, convex, and yellow colonies on nutrient agar medium.

The biochemical characters of the strains are shown in Tables 1 and 6 . They hydrolyze gelatin and esculin. They showed positive VP-reaction, and produced indole, $\beta$-galactosidase, catalase and DNase. They did not produce hydrogen sulfide, oxidase, and urease. They did not show phenylalanine deaminase, oxidation of gluconate, lysine decarboxylase, argine dihydrolase, ornithine decarboxylase, and nitrate respiration.

The fatty acid composition of strain $\mathrm{Fe}-4$ was similar to that of strain $K$. oxytoca RH 1586 as shown in Table 2. Strains Fe-3 and Fe-4 had the ubiquinone system Q-8.

The strains differed from Erwinia herbicola IAM 1562 and IAM 1584 in producing indole. However, strains Fe-3 and Fe-4 were identified as E. herbicola (Löhnis 1911) Dye 1964 on the basis of motility with peritrichous flagella, and pigmentation of colonies. They liquefied gelatin and showed DNase but did not produce urease, arginine dihydrolase, lysine decarboxylase, and ornithine decarboxylase. They did not have nitrate respiration. They did not produce acid from dulciol, adonitol, and inulin.

Klebsiella oxytoca and E. cloacae are the common nitrogen-fixing bacteria associated with a number of rice varieties $(12,13,15,52,53)$. A nitrogen-fixing $E$. herbicola strain was isolated from water and a compost of paper mill process (54), and it was found in strains preserved at the Centers for Disease Control (CDC) (Atlanta, Georgia, U.S.A.) (55). However, this is the first report of isolation of nitrogen-fixing $E$. herbicola from the roots of rice.

Source: Strains Fe-3 and Fe-4 were isolated in 1981 from Oryza sativa strain C5444. 
Klebsiella oxytoca (Flugge 1886) Lautrop 1956

Strains: OSG 5I, OG 9, NO 17, NSG 3, NG 13, and Fe-10

These strains were fermentative gram negative and non-motile rods. They formed smooth and convex colonies on solid medium. Colonies on nutrient agar plate were light brown.

Their phenotypic characters are shown in Tables 1 and 6. They produced brown pigment from gluconate medium and assimilated pectin and polygalacturonate as shown in Table 6. Strains OG 9, NSG 3, NG 13, and Fe-10 did not liquefy gelatin but strain NO $17 \mathrm{did}$. Strain OSG $5 \mathrm{I}$ liquefied gelatin at $30^{\circ} \mathrm{C}$ but not at $20^{\circ} \mathrm{C}$. Strains NG 13 and NSG 3 did not utilize hydrogen.

The fatty acid composition of the isolates was similar to that of Klebsiella oxytoca $\mathrm{RH} 1586$ as shown in Table 2 . Strain NG 13 contained a large amount of straight-chain $\mathrm{C}_{16: 0}, \mathrm{C}_{18: 1}$, and 3-OH $\mathrm{C}_{14: 0}$ acids. Like $K$. oxytoca $\mathrm{RH} 1586$ strain NG 13 had the ubiquinone system Q-8.

Consequently, all of the strains were identified as Klebsiella oxytoca (Flugge 1886) Lautrop 1956 on the basis of positive reactions for indole, hydrolysis of urea, $\beta$-galactosidase, lysine decarboxylase, and production of pigment from gluconate medium, and negative reactions for arginine dihydrolase and ornithine decarboxylase, assimilation of polygalacturonate, and production of acid from a number of sugars.

Klebsiella pneumoniae CDC 2551-63 was used as a reference strain for a nitrogen-fixing Klebsiella strain in this study. Strain K. pneumoniae CDC 2551-63 differed from strain $K$. pneumoniae IAM $12351^{\mathrm{T}}$ in the production of indole, pigmentation on gluconate medium, production of acid from inulin and polygalacturonate as shown in Table 6. On the other hand, strain K. pneumoniae CDC 255163 was similar to the isolates in the characters mentioned above, although it did not liquefy gelatin at 20 and $30^{\circ} \mathrm{C}$. Further detailed examination of the characters of strain CDC 2551-63 is needed for taxonomy in the future.

Strains Klebsiella oxytoca RH 1586 and K. pneumoniae IAM 12351 did not fix nitrogen.

Source: Strains OSG 5I, OG 9, NO 17, NSG 3, and NG 13 were isolated from paddy soil. Strain Fe-10 was isolated in 1981 from a root of Oryza sativa strain T65.

Group 4. Gram negative, spiral-shaped bacteria

Strains: SS-18, SS-20, SS-22, SS-25, SS-28, SS-29, and SS-32

These strains had spiral-shaped cells as shown in Fig. 1D. The cells were 0.5 to $1.0 \mu \mathrm{m}$ by 1.0 to $3.0 \mu \mathrm{m}$. They were gram negative. They had a polar flagellum and showed Spirillum-like motility. They did not accumulate PHB granules. Colonies were opaque, convex, and light brown plaques on nutrient agar plate.

The strains did not produce acid from glucose oxidatively and fermentatively. Phenotypic characters are shown in Table 1. Production of acid from carbon compounds is shown in Table 7. Strains SS-25, SS-28, and SS-29 were different from the other strains in the production of acid from ribose (SS-28), sorbitol (SS-29), and 


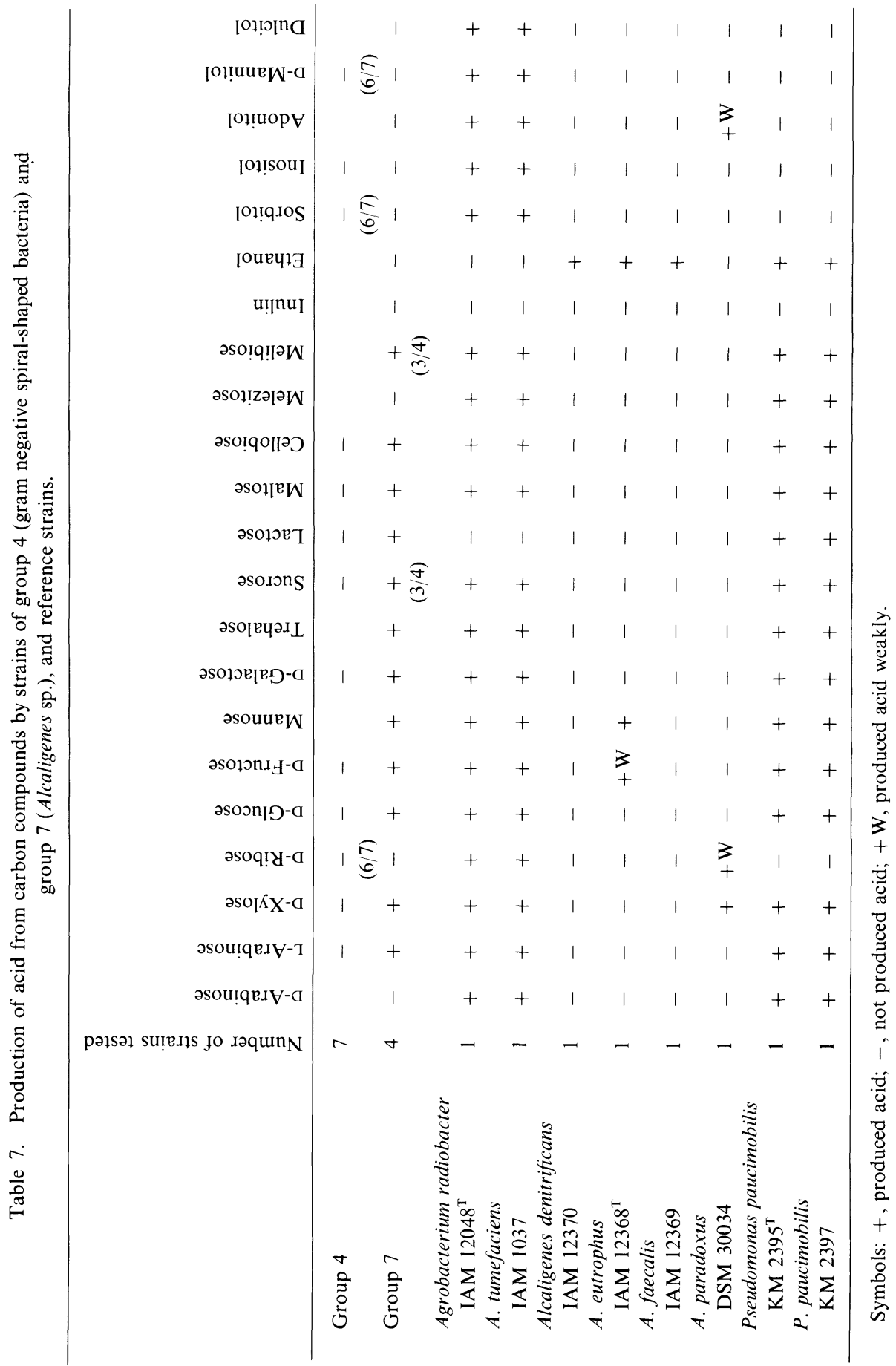


mannitol (SS-25).

The chemotaxonomic characters of the strains are shown in Table 2. The GC content of strains studied ranged from 63.1 to $65.3 \%$. They had the ubiquinone system Q-8. They had straight-chain $\mathrm{C}_{16: 0}, \mathrm{C}_{16: 1}$, and $\mathrm{C}_{18: 1}$ acids as major components, and 2-OH $\mathrm{C}_{14: 0}$ acid.

Although the strains had spiral-shaped cells and fixed nitrogen, they differed from the known species of the genus Azospirillum. The cells of the strains are smaller than those of the genus Azospirillum. The strains did not reduce nitrate, did not hydrolyze esculin, and did not produce urease and phosphatase, but Azospirillum species did. Further, their chemotaxonomic characters indicate that this group is different from the genus Azospirillum as shown in Table 2. Their GC contents were also $5 \%$ lower than those of Azospirillum brasilense IAM $12400^{\mathrm{T}}$ and $A$. lipoferum IAM $12399^{\mathrm{T}}$. The ubiquinone systems and the fatty acid composition of the strains were clearly different from those of $A$. brasilense IAM $12400^{\mathrm{T}}$ and $A$. lipoferum IAM $12399^{\mathrm{T}}$.

Source: Strains SS-18, SS-29, and SS-32 were isolated in 1982 from the roots of Oryza sativa strain T65. Strains SS-20, SS-22, and SS-25 were isolated in 1982 from the roots of Oryza sativa strain C5444.

Group 5. Gram negative pleomorphic bacteria

Strains: B-26, B-40, B-45, B-46, B-47, and B-51

These strains produced branched or pleomorphic, gram negative cells on agar and semisolid media as shown in Figs. 5A, 5B, and 5D. Old cultures showed many ovoid cells. Cells were 0.5 to $1.0 \mu \mathrm{m}$ by 1.0 to $5.0 \mu \mathrm{m}$. Nonmotile. PHB granules were accumulated in the cells. Neither water soluble nor water insoluble pigments were produced.

None of the strains produced acid from glucose oxidatively and fermentatively. Their phenotypic characters are shown in Table 1. All of the strains grew at the expense of a number of carbon compounds, especially sugars (Table 4). Strains B26, B-45, and B-47 utilized hydrogen.

The GC content of the strains ranged from 59.9 to $62.2 \%$. The strains had the ubiquinone system Q-10. They had straight-chain $C_{16: 0}, C_{16: 1}$, and $C_{18: 1}$ acids as major components, and 3-OH $\mathrm{C}_{14: 0}, 3-\mathrm{OH} \mathrm{C}_{16: 0}, 3-\mathrm{OH} \mathrm{C}_{18: 0}$ acids as shown in Table 2.

From these findings this group apparently belongs to Xanthobacter. However, they were different from Xanthobacter strains in the formation of colorless colonies, nitrate reduction, production of urease and DNase, and assimilation of sugars. They grew at the expense of L-arabinose, D-xylose, D-ribose, L-rhamnose, D-glucose, D-fructose, mannose, D-galactose, trehalose, lactose, maltose, cellobiose, salicin, malonate, sorbitol, mannitol, inositol, glycerol, L-threonine, L-serine, L-arginine, Lleucine, L-lysine, L-ornithine, L-valine, L-phenylalanine, L-histidine, and L-proline, but $X$. autotrophicus DSM 431 and DSM $432^{\mathrm{T}}$ did not (Table 4). Further, their GC contents were $6 \%$ lower than those of $X$. autotrophicus DSM 431 and DSM $432^{\mathrm{T}}$ 

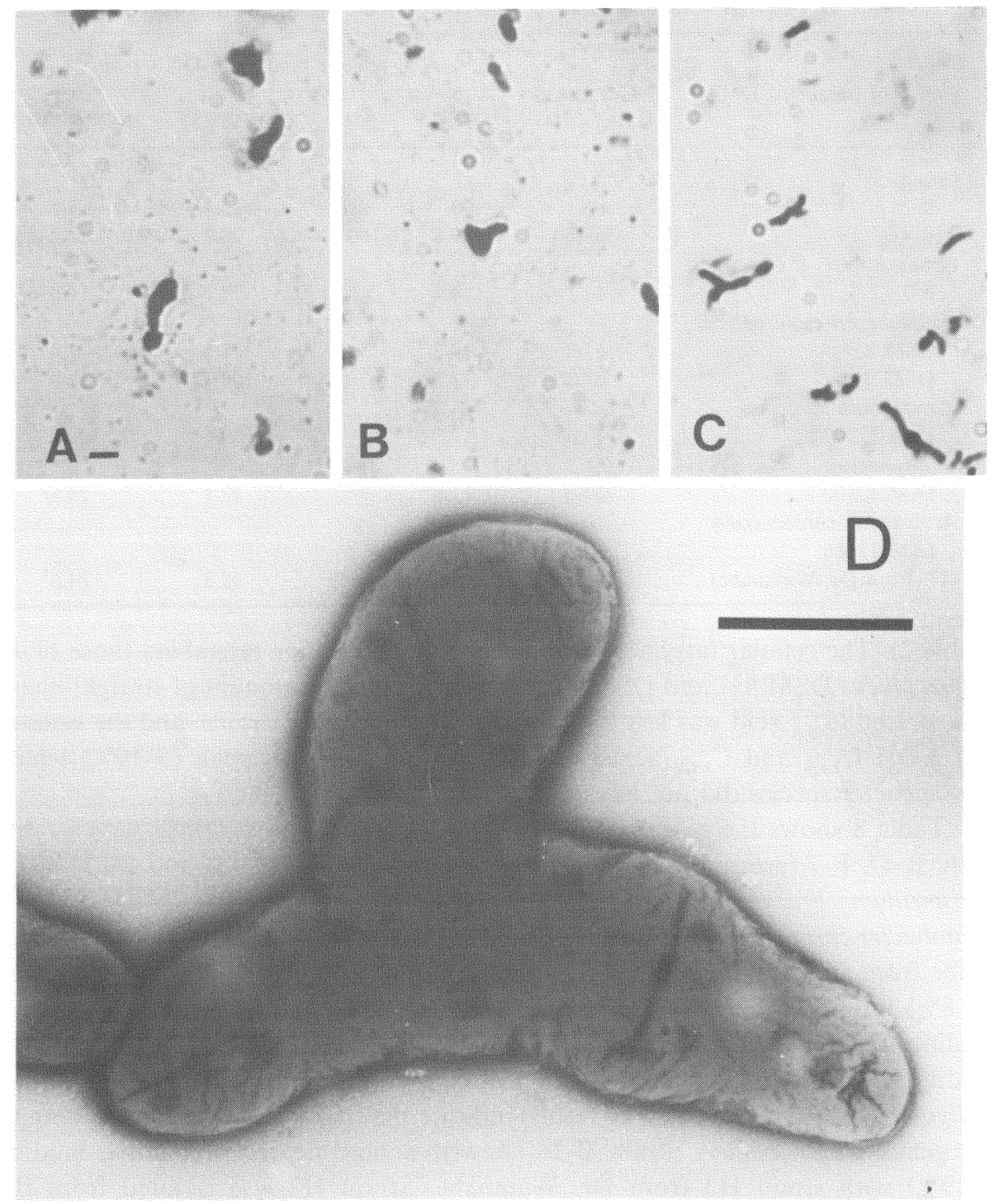

Fig. 5. Photomicrographs $(\mathrm{A}-\mathrm{C})$ and an electron micrograph (D) of strains of groups 5 (gram negative pleomorphic bacteria) and 6 (gram negative fermentative pleomorphic bacteria).

These cells were grown on nutrient agar medium for 1 day at $30^{\circ} \mathrm{C}$. A and B: strain B46 (group 5) stained with crystal violet. C: strain B-2 (group 6) stained with crystal violet. D: strain B-46 negative stain, electron microscopy. Bars indicate $1 \mu \mathrm{m}$. 
Table 8. DNA-DNA homologies among strains of group 5 and 6 and related organisms.

\begin{tabular}{|c|c|c|c|c|c|}
\hline \multirow{2}{*}{ Source of unlabeled DNA } & \multirow{2}{*}{$\mathrm{GC} \mathrm{mol} \%$} & \multicolumn{4}{|c|}{$\%$ relative binding of DNA from } \\
\hline & & B-26 & B-47 & DSM 432 & IAM 12399 \\
\hline \multicolumn{6}{|l|}{ Group 5} \\
\hline B-26 & 61.1 & 100 & 98.1 & 12.1 & 9.1 \\
\hline B-47 & 62.2 & 99.8 & 100 & 20.7 & 14.2 \\
\hline \multicolumn{6}{|l|}{ Group 6} \\
\hline $\mathrm{F}-7$ & 63.1 & 4.4 & & 28.7 & 14.9 \\
\hline \multicolumn{6}{|l|}{ Xanthobacter autotrophicus } \\
\hline DSM 431 & 67.2 & 14.2 & & 75.8 & 7.4 \\
\hline $\operatorname{DSM} 432^{\mathrm{T}}$ & 67.0 & 3.3 & & 100 & 20.0 \\
\hline \multicolumn{6}{|l|}{ Azospirillum lipoferum } \\
\hline IAM $12399^{\mathrm{T}}$ & 69.0 & 17.3 & & 30.5 & 100 \\
\hline \multicolumn{6}{|l|}{ A. brassilense } \\
\hline IAM 12400 & 69.0 & 17.8 & & 22.1 & 24.6 \\
\hline \multicolumn{6}{|l|}{ Azotobacter chroococcum } \\
\hline IAM 12666 & & 7.3 & & & \\
\hline H3 (from Dr. Watanabe) & 61.1 & 99.9 & & 48.3 & 24.6 \\
\hline
\end{tabular}

(Table 2). The cellular fatty acid composition of the strains resembled those of $X$. autotrophicus DSM 431 and DSM $432^{\mathrm{T}}$ (Table 2). But the amount of straight-chain unsaturated $\mathrm{C}_{18: 1}$ acid was less than those of Xanthobacter strains, and the isolates had 3-OH $\mathrm{C}_{14: 0}$ and $\mathrm{C}_{18: 0}$ acids, and showed an unknown peak (5-10\%) which Xanthobacter species did not have.

Table 8 shows the results of DNA-DNA hybridization obtained with strains B-26, B-47, F-7 (group 6), Xanthobacter autotrophicus DSM 431 and DSM 432 ${ }^{\mathrm{T}}$, Azospirillum brasilense IAM $12400^{\mathrm{T}}$, Azospirillum lipoferum IAM $12399^{\mathrm{T}}$, Azotobacter chroococcum IAM $12666^{\mathrm{T}}$, and $\mathrm{H} 3$ from Dr. Watanabe. With the DNA probe from strain B-26, strain B-47 showed a very high degree of relative binding $(99.8 \%)$ but the other strains, except strain $\mathrm{H} 3$, showed low degrees of relative binding (3.3 to $17.3 \%$ ). Strains B-26 and B-47 showed low degrees of relative binding ( 14.2 to $28.7 \%$ ) with the DNA probes from $X$. autotrophicus DSM $432^{\mathrm{T}}$ and A. lipoferum IAM $12399^{\mathrm{T}}$. These results indicate that these strains do not belong to the genus Xanthobacter. Strain B-26 showed a high degree of relative binding $(99.9 \%$ ) with strain H3 from Dr. Watanabe. Strain H3 was isolated from the rhizosphere of rice, and identified as Pseudomonas sp. by BARraQuio et al. (17).

Source: Strain B-26 was isolated in 1982 from the roots of Oryza sativa strain T65. Strains B-46 and B-47 were isolated in 1981 from the roots of Oryza sativa strain T65. Strains B-40 and B-45 were isolated in 1981 from the roots of Oryza sativa strain C5444. Strain B-51 was in 1982 from the roots of Oryza sativa strain C5444.

Group 6. Gram negative fermentative pleomorphic bacteria Strains: F-4, F-7, B-2, B-4, B-7, B-18, B-25, and B-32 
The strains studied resembled the group 5 strains in many respects. They produced nonmotile, gram negative, pleomorphic cells as shown in Fig. 5C. The cells were 0.5 to $1.0 \mu \mathrm{m}$ by 1.0 to $5.0 \mu \mathrm{m}$. Old cells became ovoid as the group 5 strains did. PHB granules were accumulated in cells. Neither water-soluble nor water-insoluble pigments were produced.

All of strains produced acid from glucose fermentatively. Their phenotypic characters are shown in Table 1 . The strains did not hydrolyze gelatin, starch, and esculin. They did not grow on tellurite agar medium. The indole reaction was positive. They reduced nitrate but not tetrazolium chloride. They produced catalase, oxidase, urease, and tyrosinase but not phosphatase and DNase. They assimilated a number of carbon compounds (Table 4). Strains F-4, B-2, and B-32 did not utilize hydrogen.

The chemotaxonomic characters of the strains are shown in Table 2. The GC content of the strains ranged from 62.1 to $63.1 \%$. They had the ubiquinone system Q-10. They had straight-chain $\mathrm{C}_{16: 0}, \mathrm{C}_{16: 1}$, and $\mathrm{C}_{18: 1}$ acids as major compounds, and 3-OH $\mathrm{C}_{14: 0}, 3-\mathrm{OH} \mathrm{C} \mathrm{C}_{16: 0}$, and 3-OH $\mathrm{C}_{18: 0}$ acids as did the strains of group 5.

Although they had pleomorphic cells, they were different from Xanthobacter in the formation of colorless colonies, production of acid from glucose by fermentative metabolism, hydrogen utilization, and nitrate reduction. They produced indole, hydrolyzed urea, and decomposed tyrosine. They did not reduce tetrazolium chloride and did not produce phosphatase. The average value of the GC contents of the strains was $4 \%$ lower than that of $X$. autotrophicus.

Group 6 was different from group 5 in the reduction of nitrate and tetrazolium chloride, and production of indole, phosphatase and DNase. The strains did not assimilate maltose, succinate, malonate, glutamate, 2-oxoglutarate, citrate, DLlactate, $\beta$-hydroxybutyrate, $n$-propanol, methanol, ethanol, $\beta$-alanine, L-threonine, L-arginine, L-leucine, L-lysine, L-ornithine, L-valine, L-phenylalanine, or L-histidine, but the group 5 strains did. Oxalate was assimilated by the strains of this group but not by the group 5 strains.

Strain F-7 showed a low degree of relative binding with the DNA probes from strain B-26 (group 5) $(4.4 \%)$, X. autotrophicus DSM $432^{\mathrm{T}}(28.7 \%)$, and $A$. lipoferum IAM $12399^{\mathrm{T}}\left(14.9^{\%}\right)($ Table 8$)$.

Source: Strains F-4, F-7, B-2, B-4, B-7, and B-18 were isolated in 1982 from the roots of Oryza sativa strain C5444. Strains B-25 and B-32 were isolated in 1982 from the roots of Oryza sativa strain T65.

\section{Group 7. Alcaligenes sp.}

Strains: OSG 47, Y-22, Y-23, and Y-39

Small rod-shaped cells measuring 0.5 to $1.0 \mu \mathrm{m}$ by 1.0 to $3.0 \mu \mathrm{m}$ were produced on agar and semisolid media. Four strains of this group were gram negative, nonspore forming, and motile with peritrichous flagella as shown in Fig. 4D. PHB granules were accumulated in the cells. Colonies were circular, smooth, convex, entire, opaque, and yellow-orange on agar medium. 


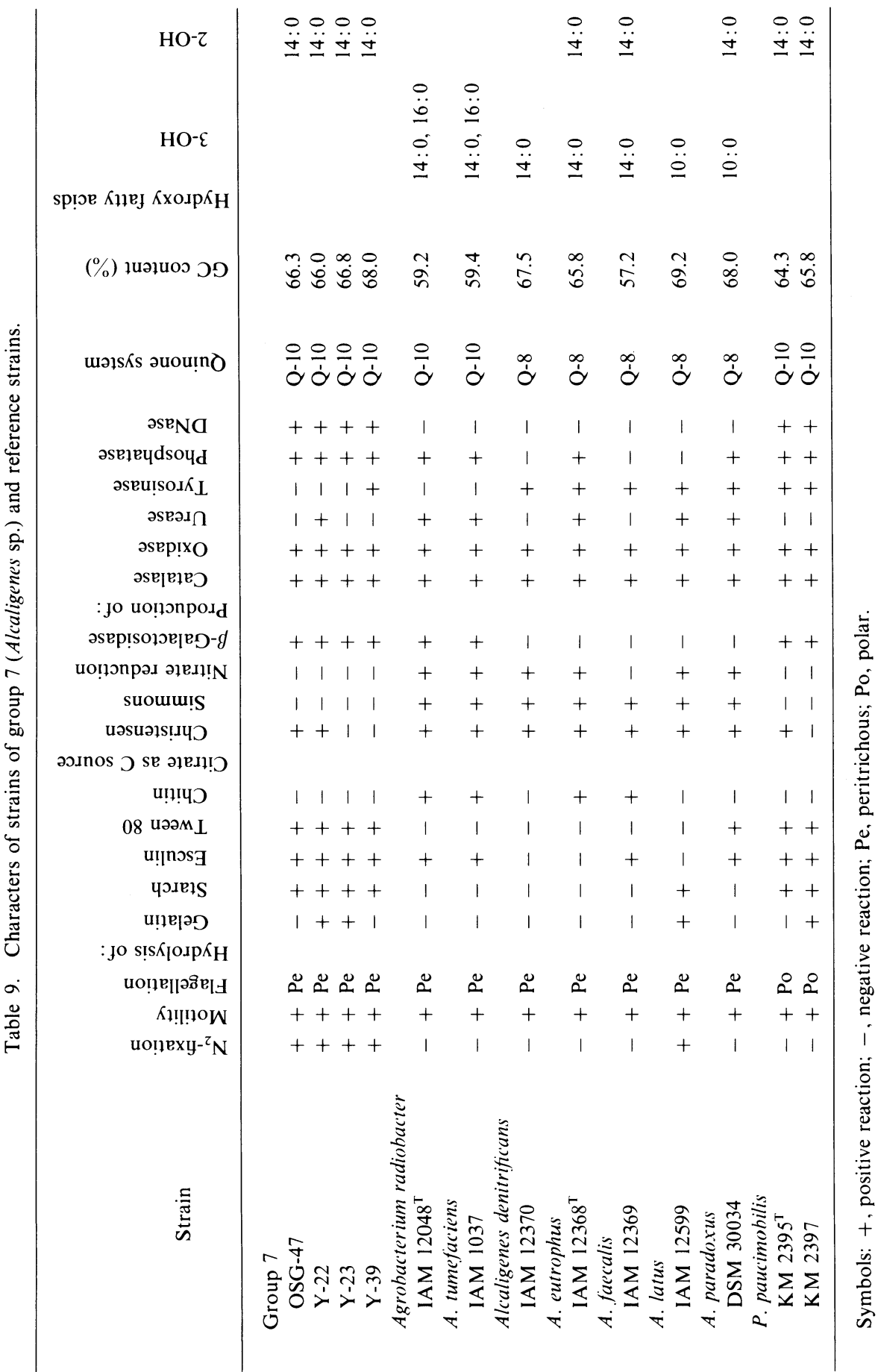


As shown in Table 9, these strains hydrolyzed starch, esculin, and Tween 80 but not chitin. They produced catalase, $\beta$-galactosidase, oxidase, phosphatase, and DNase but not produce indole, $\mathrm{H}_{2} \mathrm{~S}$, and arginine dihydrolase. They did not utilize citrate on Simmons medium and malonate. They did not reduce nitrate. Although not listed in Table 9, all isolates grew at 25,30 , and $37^{\circ} \mathrm{C}$ but not grow at $42^{\circ} \mathrm{C}$ and in $2.5,5,7.5$, and $10 \% \mathrm{NaCl}$. They did not produce 3-ketolactose from lactose. The responses to hydrolysis of gelatin, utilization of citrate on Christensen medium, production of urease and tyrosinase differed among stains as shown in Table 9. Acid was produced from L-arabinose, D-xylose, D-glucose, D-fructose, mannose, D-galactose, trehalose, lactose, maltose, cellobiose, and melibiose, but not from D-arabinose, D-ribose, melezitose, inulin, ethanol, sorbitol, inositol, adonitol, D-mannitol, and dulcitol (Table 7). Strain Y-22 did not produce acid from sucrose and melibiose but the others did. The assimilation of 40 carbon compounds is shown in Table 4. All strains grew at the expense of L-arabinose, D-xylose, Dglucose, D-fructose, trehalose, sucrose, lactose, maltose, cellobiose, raffinose, starch, acetate, succinate, DL-malate, pyruvate, DL- $\beta$-hydroxybutyrate, L-glutamine, and Laspartate, but not at the expense of malonate, adipate, citrate, DL-tartrate, DL-mandelate, gluconate, 2-ketogluconate, itaconate, methanol, glycerol, benzoate, mucate, phenol, $m$-hydroxybenzoate, betaine, $\beta$-alanine, $\beta$-aminobutyrate, and Lproline. Utilization of the other five carbon compounds, i.e., pimelate, suberate, citrate, gluconate, and ethanol differed among the strains. Strain Y-22 assimilated citrate weakly. Strain Y-23 did not assimilate suberate and ethanol. Strain Y-39 assimilated gluconate but did not pimelate. Strain OSG 47 did not utilize hydrogen.

The GC content of the strains ranged from 66.0 to $68.0 \%$, and all strains of this group had the ubiquinone system Q-10 (Table 2). They had straight-chain $\mathrm{C}_{16: 0}$, $\mathrm{C}_{16: 1}, \mathrm{C}_{18: 1}$ acids as major components, and 2-OH $\mathrm{C}_{14: 0}$ acid was found but there were no 3-hydroxy acids. Strains OSG 47 and Y-22 had sphingolipid as shown in Fig. 6. The visible absorption spectrum of acetone extract of yellow pigment had 2 peaks in 452 and $480 \mathrm{~nm}$ (Fig. 7).

The strains resemble the members in the genus Alcaligenes in having gram negative rod-shaped cells with peritrichous flagella (56), but they were different from the genus Alcaligenes in nitrate reduction, assimilation patterns of carbon compounds (57), quinone system, and fatty acid composition. Alcaligenes species had Q8 and straight-chain $\mathrm{C}_{16: 0}, \mathrm{C}_{16: 1}$, and $\mathrm{C}_{18: 1}$ acids and cyclopropane acids of $\mathrm{C}_{17: 0}$ and $\mathrm{C}_{19: 0}$ as major components and contained $3-\mathrm{OH} \mathrm{C}_{10: 0}$ or $3-\mathrm{OH} \mathrm{C}_{14: 0}$ acids (Table 2).

In the assimilation of sugars the isolates seem to be close to the members of the genus Agrobacterium, which are motile with peritrichous flagella and are well known as plant pathogens(58). Agrobacterium radiobacter IAM $12048^{\mathrm{T}}$ and $A$. tumefaciens IAM 1037 had Q-10 (Table 2). However, the isolates did not produce 3ketolactose from lactose but $A$. radiobacter IAM $12048^{\mathrm{T}}$ and $A$. tumefaciens IAM $1037 \mathrm{did}$. The isolates did not assimilate gluconate and 2-ketogluconate but $A$. radiobacter IAM $12048^{\mathrm{T}}$ and $A$. tumefaciens IAM $1037 \mathrm{did}$. The GC content of 


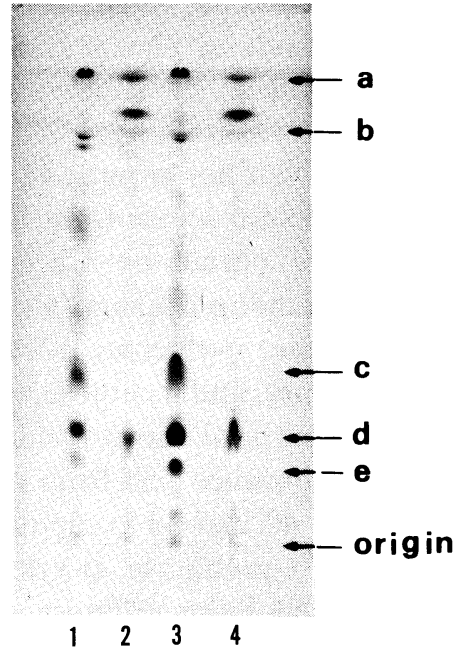

Fig. 6.

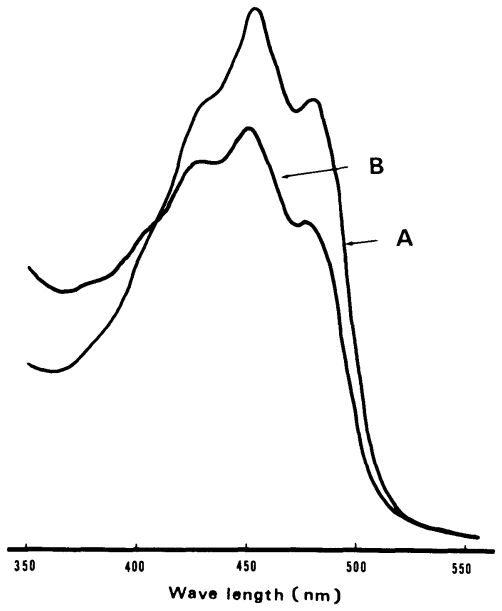

Fig. 7.

Fig. 6. Thin-layer chromatography of original and alkaline stable lipid from $P$. paucimobilis $\mathrm{KM} 2395^{\mathrm{T}}$ and strain OSG 47.

(1) Original lipid from $P$. paucimobilis $\mathrm{KM} 2395^{\mathrm{T}}$, (2) Lipid obtained after alkaline hydrolysis $(0.5 \mathrm{~N} \mathrm{KOH}$ methanol-chloroform $(1: 1$ by vol.) for $6 \mathrm{~h}$ at room temperature) from $P$. paucimobilis KM 2395, (3) Original lipid from strain OSG 47, (4) Lipid obtained after alkaline hydrolysis from strain OSG 47. a, fatty acids; b, unknown lipid; c, unknown phospholipid; d, unknown glycolipid (alkaline stable); e, unknown phospholipid. TLC solvent: chloroform-methanol-acetic acid-water (100:20:12:5, by vol.).

Fig. 7. Visible absorption spectra of yellow pigments in acetone-extract.

A: strain OSG 47 (group 7). B: Pseudomonas paucimobilis KM 2395 ${ }^{\mathrm{T}}$.

Agrobacterium radiobacter IAM $12048^{\mathrm{T}}(59.2 \%)$ and $A$. tumefaciens IAM 1037 $(59.4 \%$ ) was $7 \%$ lower than the isolates. The fatty acid composition of Agrobacterium differed from those of the isolates (Table 2). Agrobacterium contained straight-chain $\mathrm{C}_{16: 0}$ and cyclopropane of $\mathrm{C}_{19: 0}$ acids as major components, and 3-OH $\mathrm{C}_{14: 0}$ and 3-OH $\mathrm{C}_{10: 0}$ acids but not 2-hydroxy acid.

On the other hand, the isolates closely resemble Pseudomonas paucimobilis in phenotypic and chemotaxonomic characters except for their flagellation, acid production from arabinose and ethanol, and assimilation of galactose. The strains had PHB granules in the cells and the same visible absorption spectrum of yellow pigments in acetone-extract as $P$. paucimobilis KM $2395^{\mathrm{T}}$ (Fig. 7). They hydrolyzed starch, esculin, and Tween 80 , produced phosphatase and DNase, as $P$. paucimobilis did. Also, the isolates had only 2-OH C $\mathrm{C}_{14: 0}$ acid and did not contain 3-hydroxy acid as $P$. paucimobilis did (59-61). The strains had sphingolipid (Fig. 6) and ubiquinone system Q-10, as $P$. paucimobilis did. Table 10 shows the DNA-DNA hybridization between isolates (OSG 47 and Y-39), P. paucimobilis KM 2395 $5^{\mathrm{T}}$ and KM 2397, Alcaligenes faecalis IAM 12369, Alcaligenes latus IAM 12599, Agrobacterium 
Table 10. DNA-DNA homologies among strains of group 7 (Alcaligenes sp.) and related organisms.

\begin{tabular}{|c|c|c|c|c|}
\hline \multirow{2}{*}{ Source of unlabeled DNA } & \multirow{2}{*}{$\mathrm{GC} \mathrm{mol} \%$} & \multicolumn{3}{|c|}{$\%$ relative binding of DNA from } \\
\hline & & OSG 47 & KM 2397 & IAM 12369 \\
\hline \multicolumn{5}{|l|}{ Group 7} \\
\hline OSG 47 & 66.3 & 100 & 24.8 & 1.7 \\
\hline Y-39 & 68.0 & 99.7 & 19.2 & 2.6 \\
\hline \multicolumn{5}{|l|}{ Pseudomonas paucimobilis } \\
\hline KM $2395^{\mathrm{T}}$ & 64.3 & 55.7 & 92.9 & 3.8 \\
\hline KM 2397 & 65.8 & 34.0 & 100 & 2.7 \\
\hline \multicolumn{5}{|l|}{ Alcaligenes faecalis } \\
\hline IAM 12369 & 57.2 & 3.3 & 1.6 & 100 \\
\hline \multicolumn{5}{|l|}{ A. latus } \\
\hline IAM 12599 & 69.2 & 1.0 & 2.1 & 1.0 \\
\hline \multicolumn{5}{|l|}{ Agrobacterium tumefaciens } \\
\hline IAM 1037 & 59.4 & 18.3 & 9.1 & 5.3 \\
\hline \multicolumn{5}{|l|}{ Azospirillum lipoferum } \\
\hline IAM $12399^{\mathrm{T}}$ & 69.0 & 23.3 & 5.2 & 2.9 \\
\hline H3 from Dr. Watanabe & 61.1 & 13.4 & & \\
\hline
\end{tabular}

tumefaciens IAM 1037, Azospirillum lipoferum IAM $12399^{\mathrm{T}}$, and strain $\mathrm{H} 3$. The degree of relative binding between strains OSG 47 and Y-39 was high $(99.7 \%)$. With the DNA probe from strain OSG 47, P. paucimobilis KM 2395 and KM 2397 showed relatively high degrees of the relative binding $(34.0-55.7 \%)$, but the other five strains gave low degrees of the relative binding (1.0-23.3\%). Isolates showed 19.2-24.8\% relative bindings with the DNA probe from P. paucimobilis KM 2397. With respect to the DNA-DNA hybridization, the isolates are not the same species as $P$. paucimobilis but appeared to be very close to $P$. paucimobilis.

Recently nitrogen-fixing $P$. paucimobilis was isolated from rice $(12,13)$, Deschampsia caespitosa (62), and soil (63). The acetylene reduction activity of the type strain of $P$. paucimobilis KM $2395^{\mathrm{T}}$ was tested but no activity was found.

Source: Strains OSG 47 and Y-22 were isolated from paddy soil. Strains Y-23 and Y-39 were isolated in 1982 from the roots of Oryza sativa strain C5444.

Group 8. Protomonas-like bacteria

Strains: R-2, R-4, R-5, R-7, R-11, R-12, R-13, and R-15

All strains of group 8 were gram negative, motile with single polar flagella (Fig. 4B). The cells were 0.5 to $1.0 \mu \mathrm{m}$ by 1.0 to $3.0 \mu \mathrm{m}$. PHB granules were not accumulated in the cells. Colonies were circular, smooth, convex, entire, opaque and red on agar medium. The strains studied did not produce fluorescent and water soluble pigments in King A and B media.

Acid was not produced from glucose oxidatively and fermentatively. Strains studied did not grow in more than $2.5 \% \mathrm{NaCl}$ and at $42^{\circ} \mathrm{C}$. They hydrolyzed Tween 
80 but did not hydrolyze gelatin, starch, and esculin. Nitrate reduction and production of tyrosinase, phosphatase, and DNase were negative but tetrazolium chloride reduction (TTC reduction) and production of oxidase, catalase, and urease were positive. Table 4 shows the results of assimilation of 33 carbon compounds by isolates. Strains studied assimilated acetate (weakly), oxalate (weakly), succinate, malonate (weakly), glutarate, glyoxylate, propionate (weakly), $n$-propanol, methanol (weakly), ethanol, formaldehyde, ethylamine, glycine (weakly), and $\beta$ alanine but not sugars. Strains R-2, R-5, and R-12 did not utilize hydrogen.

The GC content of the strains ranged from 62.2 to $65.1 \%$ and, they had ubiquinone system Q-10. They had straight-chain fatty acids and straight-chain hydroxy fatty acid of 3-OH $\mathrm{C}_{14: 0}$ (Table 2).

Urakami and Komagata (64) proposed the genus Protomonas, for pinkpigmented facultatively methylotrophic bacteria that produce carotenoids and bacteriochlorophyll a in the cells grown aerobically in intermittent light. On the basis of presence of the red pigment, ubiquinone system Q-10, and fatty acid composition, isolates seem to be closely related to this genus. However, the GC content of the isolates ranged from 61.2 to $62.3 \%$, and were $5 \%$ lower than that of Protomonas. Isolates had straight-chain $\mathrm{C}_{12: 0}, \mathrm{C}_{14: 0}, \mathrm{C}_{19: 0}$ and cyclopropane of $\mathrm{C}_{17: 0}$ acids but $P$. extroquens $\mathrm{TK} 0001^{\mathrm{T}}$ did not, and the amounts of straight-chain $\mathrm{C}_{16: 0}$, $\mathrm{C}_{16: 1}, \mathrm{C}_{18: 0}$, and $\mathrm{C}_{18: 1}$ acids were different from those of $P$. extroquens. Further, the isolates did not assimilate DL-lactate, DL-tartrate, formate, formamide, and methylamine, and did not accumulate PHB granules. They assimilated methanol very weakly compared to P. extroquens TK $0001^{\mathrm{T}}$. Protomonas extroquens TK $0001^{\mathrm{T}} \mathrm{did}$ not fix nitrogen.

Some pink-pigmented bacteria motile with subpolar flagella, and having 60 $64 \%$ GC contents and the ubiquinone system Q-10 were studied by SHIBA and SHIMIDZU (65), and for those organisms a new genus Erythrobacter was established. However their habitat is marine environments.

Source: Strains R-2, R-4, R-7, and R-11 were isolated in 1982 from the roots of Oryza sativa strain T65. Strains R-12 and R-15 were isolated in 1981 from the roots of Oryza sativa strain T65. Strain R-5 was isolated in 1982 from the roots of Oryza sativa strain C5444. Strain R-13 was isolated in 1981 from the roots of Oryza sativa strain C5444.

Group 9. Azotobacter-like bacteria

Strains: A-2, A-3, A-4, A-7, and A-24

These strains were gram negative and motile with a polar flagellum as shown in Fig. 4C. The cells were 0.5 to $0.7 \mu \mathrm{m}$ by 1.5 to $2.0 \mu \mathrm{m}$. Old cells became ovoid. They did not form cysts. They grew slowly and produced characteristic wrinkled hard colonies on agar medium, but did not produce viscid slime and water soluble fluorescent pigments in King A and B media and in mannitol yeast-extract medium. They accumulated PHB granules in their cells.

They did not hydrolyze gelatin, starch, or Tween 80 . They reduced nitrate. 
Acid was not produced from glucose oxidatively and fermentatively. Catalase and oxidase were produced but DNase was not. Strains A-2, A-3, and A-7 did not utilize hydrogen.

The GC content of the strains studied ranged from 64.1 to $65.1 \%$ as shown in Table 2. They had the ubiquinone system Q-10 and straight-chain $\mathrm{C}_{16: 0}, \mathrm{C}_{16: 1}$, and $\mathrm{C}_{18: 1}$ acids as major components, and $2-\mathrm{OH} \mathrm{C} \mathrm{C}_{14: 0}$ and $3-\mathrm{OH} \mathrm{C}_{12: 0}$ acids as minor components.

The isolates were considered to belong to the Azotobacteriaceae in cell size and the presence of the PHB granules in the cells, and the cells becoming ovoid. The isolates appear to be related to the genus Azotobacter or Derxia in their ubiquinone system Q-8, straight-chain $\mathrm{C}_{16: 0}, \mathrm{C}_{16: 1}$, and $\mathrm{C}_{18: 1}$ acids as major components, and 3$\mathrm{OH} \mathrm{C} \mathrm{C}_{12: 0}$ and 2-OH C $\mathrm{C}_{14: 0}$ acids as minor components. The $\mathrm{GC}$ contents of the strains of Azotobacteriaceae were reported by DE LEY (66) to be $64.8-66.5 \%$ for Azotobacter, 57-58.6\% for Azomonas, 54.7-59\% for Beijerinkia, $70 \%$ for Derxia. The isolates resemble the genus Derxia more than the genus Azotobacter in their polar flagellation, but are different from in the reaction of catalase, utilization of hydrogen, and GC content. However, the isolates were different from Azotobacter in their cyst formation and flagellation.

Source: Strain A-2 was isolated in 1982 from the roots of Oryza sativa strain T65. Strains A-3, A-4, A-7, and A-24 were isolated in 1982 from the roots of Oryza sativa strain C5444.

Group 10. Others

Strains: O-1, O-3, O-5, O-6, O-9, O-11, O-15, O-16, and OSG 53III

The nine strains of this group were different from those of the other nine groups in biochemical and chemotaxonomic characters. All of these strains were gram negative rods and did not produce fluorescent pigments in King A and B media. Some strains hydrolyzed starch (O-3) and Tween 80 (O-5 and OSG 53III) but others did not. Some strains produced tyrosinase (O-6 and OSG 53III) and DNase (O-3 and OSG 53III) but others did not. Some strains did not produce urease (O-3 and OSG 53III) but others did. Strains O-3, O-6, O-9, and O-11 had the ubiquinone system Q-8 and OSG 53III had the ubiquinone system Q-10.

For the identification of these strains more precise characterization is needed.

Source: Strains O-1, O-6, O-9, and O-11 were isolated in 1982 from the roots of Oryza sativa strain C5444. Strains O-15 and O-16 were isolated in 1981 from the roots of Oryza sativa strain T65. Strain OSG 53III was isolated from paddy soil.

Döberiner's malate semisolid medium has been used most frequently to isolate nitrogen-fixing bacteria from plants and soil $(2,4,8,9,11-14,18,67-69)$. The most frequently isolated nitrogen-fixing bacteria in this medium were species of Azospirillum. This uniformity seems to reflect the carbon source (malate) of selective medium and semisolid agar culture. Nevertheless, a large number and many kinds of nitrogen-fixing bacteria were isolated from the rhizosphere of rice and it was 
found that more than $10 \%$ of the bacteria around the rhizosphere of rice fixed nitrogen (details will be reported in another paper).

Of these strains isolated from rice some were identified as Azospirillum brasilense, A. lipoferum, Enterobacter cloacae, Erwinia herbicola, Klebsiella oxytoca, and Xanthobacter autotrophicus but the others were not assigned to any known species. Unidentified strains were spirals (group 4), gram negative pleomorphic bacteria (group 5), gram negative fermentative pleomorphic bacteria (group 6), Alcaligenes sp. (group 7), Protomonas-like bacteria (group 8), Azotobacter-like bacteria (group 9), and others (group 10). These findings may suggest that the more improvement of the procedure and the selective medium for isolation advance the more unknown nitrogen-fixing bacteria will be found around the rhizosphere of rice. Using chemotaxonomic methods for identification may make clear the characters of nitrogen-fixing bacteria, as mentioned in our experiments. But there are still a large number of unidentified nitrogen-fixing bacteria. To identify them, more detailed characterization is needed.

The strains of groups 5 and 6 had pleomorphic cells, as Xanthobacter species have. Further, the strains of group 5 utilized hydrogen as Xanthobacter species did. However the strains of group 5 were different from Xanthobacter phenotypically and chemotaxonomically and in the results of the DNA-DNA hybridization. Although more detailed characterization is needed to identify those group 5 strains, they are considered to constitute a new genus. On the other hand, the strains of groups 1 and 5 utilized hydrogen. The ability to utilize hydrogen was found in $A$. lipoferum(70). WATANABE et al.(16) reported the predominance of hydrogenutilizing bacteria among nitrogen-fixing bacteria in wetland rice roots. Since a large number of nitrogen-fixing bacteria occur around the rhizosphere of rice, hydrogenutilizing and nitrogen-fixing bacteria appear to have some relationships with rice.

We thank late Dr. T. Fujii, National Institute of Genetics, Mishima, for supplying plant materials and paddy soils. We also thank Dr. J. Sugiyama, Institute of Applied Microbiology, The University of Tokyo, Tokyo, for his helpful discussion. We also thank Mrs. A. Hirata, Institute of Applied Microbiology, The University of Tokyo, Tokyo, for kindly photographing electron micrographs of the strains.

\section{REFERENCES}

1) T. Yoshida and R. R. Ancajas, Proc. Soil Sci. Soc. Am., 35, 156 (1970).

2) Y. Dommergues, J. Balandreau, G. Rinaudo, and P. Weihard, Soil Biol. Biochem., 5, 83 (1973).

3) T. Yoshida and R. R. Ancajas, Soil Biol. Biochem., 5, 153 (1973).

4) J. P. Balandreau, C. R. Millier, and Y. R. Dommergues, Appl. Environ. Microbiol., 27, 662 (1974).

5) Y. Hirota, T. Fujil, Y. Sano, and S. Iyama, Nature, 276, 416 (1978).

6) I. Watanabe and D. R. Cabrera, Appl. Environ. Microbiol., 37, 373 (1979).

7) I. Watanabe, W. L. Barraquio, M. R. de Guzman, and D. A. Cabrera, Appl. Environ. Microbiol., 37, 813 (1979).

8) M. Habte and M. Alexander, Appl. Environ. Microbiol., 40, 507 (1890).

9) J. F. Baldensperger, Plant Soil, 57, 439 (1980). 
10) A. A. App, I. Watanabe, M. Alexander, W. Ventura, C. Daez, T. Santiago, and S. K. De DatTa, Soil Sci., 130, 283 (1980).

11) M. L. Kumari, S. K. Kavimandan, and N. S. Subba-Rao, Indian J. Exp. Biol., 14, 638 (1976).

12) D. Thomas-Bauzon, P. Weinhard, T. Heulin, and J. Balabdreau, Can. J. Microbiol., 28, 922 (1982).

13) R. Bally, D. Thomas-Bauzon, T. Heulin, J. Balandreau, C. Richard, and L. De Ley, Can. J. Microbiol., 29, 881 (1983).

14) R. B. Lamm and C. A. Neyra, Can. J. Microbiol., 27, 1320 (1981).

15) J. K. Ladha, W. L. Barraquio, and I. Watanabe, Can. J. Microbiol., 29, 1301 (1983).

16) I. Watanabe, W. L. Barraquio, and M. L. Darov, Can. J. Microbiol., 28, 1051 (1982).

17) W. L. Barraquio, J. K. Ladha, and I. Watanabe, Can. J. Microbiol., 29, 867 (1983).

18) Y. Sano, T. Fujil, S. Iyama, Y. Hirota, and K. Komagata, Crop. Sci., 21, 758 (1981).

19) J. F. W. von Bulow and J. Döbereiner, Proc. Natl. Acad. Sci. U.S.A., 72, 2389 (1975).

20) R. Y. Stanier, M. Doudoroff, and E. A. Adelberg, The Microbial World, 1st ed., Prentice-Hall, Inc., Englewood Cliffs, N. J. (1957), p. 287.

21) G. J. Hucker and H. J. Conn, Tech. Bull. N. Y. St. Agric. Exp. Stn., No. 93 (1923).

22) T. Toda, Nippon Iji Shinpo, 283, 113 (1928), in Japanese.

23) A. R. Spurr, J. Ultrastruct. Res., 26, 31 (1969).

24) E. S. Reynolds, J. Cell. Biol., 17, 208 (1963).

25) K. L. Burdon, J. Bacteriol., 52, 665 (1946).

26) A. G. Ostle and G. Holt, Appl. Environ. Microbiol., 44, 238 (1982).

27) S. T. Cowan, Cowan and Steel's Manual for the Identification of Medical Bacteria, 2nd ed., Cambridge Univ. Press, Cambridge (1974), pp. 137-180.

28) L. H. Stevenson and M. D. Socolofsky, J. Bacteriol., 91, 304 (1699).

29) J. J. Tarrand, N. R. Kreig, and J. Döbereiner, Can. J. Microbiol., 24, 967 (1978).

30) H. Korth, I. Orskov, and G. Pulverer, Zentralbl. Bakteriol. Infektionskr. Hyg., Abt. 1, Orig. A, 211, 105 (1969).

31) P. B. Hylemon, J. S. Wells, Jr., N. R. Kreige, and H. W. Jannasch, Int. J. Syst. Bacteriol., 23, 340 (1973).

32) M. J. Thornley, J. Appl. Bacteriol., 23, 37 (1960).

33) K. Komagata, Nippon Nōgeikagaku Kaishi, 35, 981 (1961), in Japanese.

34) H. Iizuka and K. Komagata, J. Gen. Appl. Microbiol., 9, 73 (1963).

35) R. SAKAZAKI, Shin Saikin Baichi Gaku Koza, Kindaishuppan, Tokyo (1978), in Japanese.

36) M. L. Bernaerts and J. De Ley, Nature, 197, 406 (1963).

37) R. Y. Stanier, N. J. Palleroni, and M. Doudoroff, J. Gen. Microbiol., 13, 159 (1966).

38) T. Urakami and K. Komagata, J. Gen. Appl. Microbiol, 25, 343 (1979).

39) P. R. Edwards and W. H. EwING, Identification of Enterobacteriaceae, 3rd ed., Burgess Publ. Co., Minn. (1972).

40) K. A. Malik and D. Claus, Int. J. Syst. Bacteriol., 29, 183 (1979).

41) H. Saito and K. Miura, Biochim. Biophys. Acta, 72, 619 (1963).

42) J. Marmur and P. Doty, J. Mol. Biol., 5, 109 (1962).

43) P. W. J. Rigby, M. Deckmann, C. Rhodes, and P. Berg, J. Mol. Biol., 113, 239 (1977).

44) T. Kaneko, In Biseibutu no Kagakubunruihou, ed. by K. Komagata, Gakkai Shuppan Center, Tokyo (1983), p. 255, in Japanese.

45) Y. Yamada, K. Aida, and T. Uemura, J. Gen. Appl. Microbiol., 15, 181 (1969).

46) S. Iкemoto, K. КатоH, and K. Komagata, J. Gen. Appl. Microbiol., 24, 41 (1978).

47) A. Yamamoto, I. Yano, M. Masui, and E. YabuUchi, J. Biochem., 83, 1213 (1978).

48) E. Yabuuchi, E. Tanimura, A. Ohyama, I. Yano, and A. Yamamoto, J. Gen. Appl. Microbiol., 25, 95 (1979).

49) E. C. Falk, J. Döbereiner, J. L. Johnson, and N. R. Kreig, Int. J. Syst. Bacteriol., 35, 117 (1985). 
50) B. Reinhold, T. Hurek, I. Fendrik, B. Pot, M. Gillis, K. Kersters, S. Thielemans, and J. De LEY, Int. J. Syst. Bacteriol., 37, 43 (1987).

51) N. R. Kreig and J. Döbereiner, Genus Azospirillum, In Bergey's Manual of Systematic Bacteriology, Vol. 1, ed. by N. R. Kreig and J. G. HolT, Williams \& Wilkins, Baltimore (1986), p. 94.

52) S. A. Kulasooriya, P. A. Roger, W. L. Barraquio, and I. Watanabe, Soil Sci. Plant Nutr. (Tokyo), 27, 19 (1981).

$53)$ B. Sengupta, A. S. Nandi, R. K. Samanta, P. Pal, D. N. Sengupta, and S. P. Sen, Ann. Bot., 48, 705 (1981).

54) A. H. Neilson and L. Sparell, Appl. Environ. Microbiol., 32, 197 (1976).

55) H. Papen and D. Werner, Arch. Microbiol., 120, 25 (1979).

56) K. Kersters and J. De Ley, Genus Alcaligenes, In Bergey's Manual of Systematic Bacteriology, Vol. 1, ed. by N. R. Kreig and J. G. Holt, Williams \& Wilkins, Baltimore (1986), p. 361.

57) K. Yamasato, M. Akagawa, N. Oishi, and H. Kuraishi, J. Gen. Appl. Microbiol., 28, 195 (1982).

58) K. Kersters and J. De Ley, Genus Agrobacterium, In Bergey's Manual of Systematic Bacteriology, Vol. 1, ed. by N. R. Kreig and J. G. Holt, Williams \& Wilkins, Baltimore (1986), p. 244.

59) B. Holmes, R. J. Owen, A. Evans, H. Malnick, and W. R. Willcox, Int. J. Syst. Bacteriol., 27, 133 (1977).

60) H. Oyaizu and K. Komagata, J. Gen. Appl. Microbiol., 29, 17 (1983).

61) K. Kawahara, K. Uchida, and K. Aida, Biochim. Biophys. Acta, 712, 571 (1982).

62) K. HaAhtela, K. Kariz, and V. Sundman, Appl. Environ. Microbiol., 45, 563 (1983).

63) R. J. Rennie, Can. J. Microbiol., 27, 8 (1981).

64) T. Urakami and K. Komagata, Int. J. Syst. Bacteriol., 34, 188 (1984).

65) T. Shiba and U. Shimidzu, Int. J. Syst. Bacteriol., 32, 211 (1982).

66) J. De Ley, Antonie van Leeuwenhoek J. Microbiol. Serol., 34, 66 (1968).

67) Y. Okon, S. L. Albrecht, and R. H. Burris, Appl. Environ. Microbiol., 33, 86 (1977).

68) M. E. Tyler, J. R. Milam, R. L. Smith, S. C. Schank, and D. A. Zuberer, Can. J. Microbiol., 25, 693 (1979).

69) I. Nur, Y. Okon, and Y. Henis, Can. J. Microbiol., 26, 714 (1980).

70) P. P. Wong, N. E. Stenberg, and L. Edgar, Can. J. Microbiol., 26, 291 (1980). 